\title{
Claudia Keller
}

\section{Von der unmöglichen Möglichkeit, Oswald Egger zu lesen. Ein Protokoll}

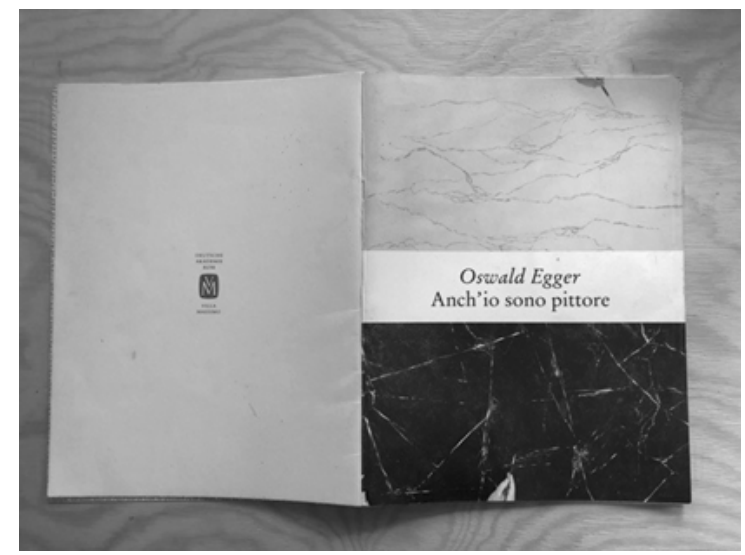

Abb. 1: Eggers Anch'io sono pittore, auf dem Schreibtisch liegend.

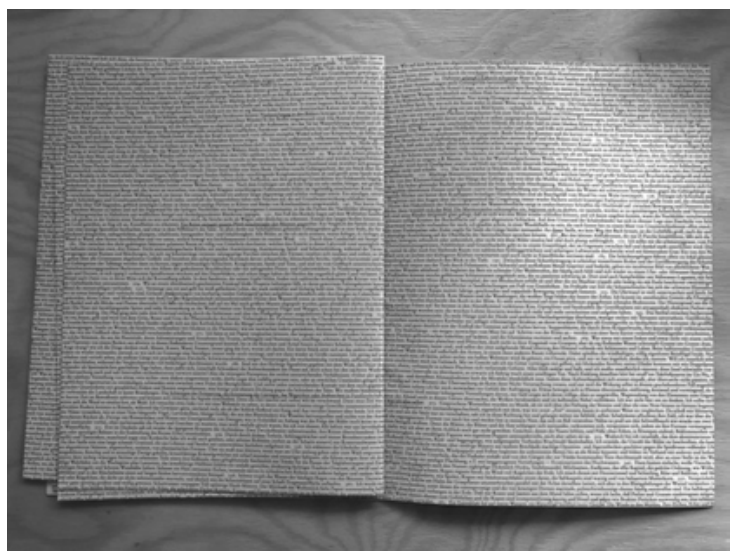

Abb. 2: Zwei Seiten des sechzehn Seiten umfassenden Textes. Zu lesen ist nicht pro Seite, sondern in der durchlaufenden Richtung einer jeweiligen Zeile quer über alle sechzehn Seiten hinweg.

Claudia Keller, Zürich 


\section{Prolog}

Über den Text, über den ich hier schreibe, sollte ich eigentlich gar nicht schreiben können. Ich habe das Heft, das die Autorbezeichnung »Oswald Egger« und den Titel "Anch'io sono pittore " trägt, über ein Antiquariat erworben. Es gibt keine ISBN-Nummer, der einzige Publikationshinweis ist das Logo der Villa Massimo der Deutschen Akademie Rom auf der Rückseite. Öffentlich zugänglich ist der Text im Jahresbericht 2014 ebendieser Institution, deren Stipendiat Egger in jenem Jahr war. ${ }^{1}$ Nach Oswald Eggers Auskunft handelt es sich um ein in Paragrafen aufgeteiltes Arbeitsjournal, um ein `kleines Diarium،, in das während seines einjährigen Aufenthalts in Rom >die Summe der täglichen, vorarbeitenden Textzellen zur weiteren Bearbeitung، eingegangen waren. ${ }^{2}$

Im Jahresbericht sind verschiedene Fotos von Lesungen abgebildet - Lesungen, die von den Festrednern gelobt (wenn auch nicht verstanden) werden. ${ }^{3}$ Am Ende des Jahresberichts kann man sehen, was die Stipendiat*innen sproduziert haben: Einblicke in die Studios, in die Notizen, Partituren, Fotografien, Texte, Entwürfe ebenso wie in der Werkstatt entstehende ,Werker. Der Text »Anch'io sono pittore" war für diesen (internen) Kontext gedacht: Erst durch meine Kontaktaufnahme wurde Egger bewusst, dass sein Journal nicht nur im gedruckten Jahresbericht, sondern dieser auch vollumfänglich im Internet einsehbar war. Und von den 50 gedruckten Exemplaren habe er etwa 10 an senge Freunder verschenkt, die anderen lägen noch bei ihm in einer Schachtel - dass ein Exemplar über ein Antiquariat den Weg gerade zu mir gefunden und ich mich dazu entschlossen hatte, darüber zu schreiben, war ein nicht kalkulierbarer Zufall. Für mich, auf deren

$1 \mathrm{https}$ //www.villamassimo.de/content/4-informationen/1-villa-massimo/3-studienaufent halt/5-jahresberichte/jahresbericht_2014.pdf (= Egger 2015a). Eggers Text wird im Folgenden mit der Sigle Asp direkt im Lauftext zitiert. Die Zitate erfolgen ohne Angabe von Seitenzahlen, da der Text unpaginiert ist. Die Publikation im Jahresbericht (Blüher 2015) kann jedoch nach Stichworten durchsucht werden.

2 Oswald Egger und ich haben uns am 3. September 2020 per E-Mail und telefonisch ausgetauscht. Ich danke Oswald Egger für die Bereitschaft, mit mir ins Gespräch zu kommen. Im folgenden Text sind aus dem Gedächtnis zitierte Nachweise aus dem Gespräch mit einfachen Anführungsstrichen markiert, Zitate aus dem Mailwechsel mit doppelten.

3 Blüher 2015, 103-106: „Ganz großen Applaus bekam an diesem Abend aber der, der die Rede der Stipendiaten hielt: Oswald Egger. Seine ungewöhnliche Art (die ich jedenfalls nicht in Worte fassen kann) hatte eine Festrede zur Folge, die keine Festrede war. Bei dieser Gelegenheit räumte er mit allem auf, was zu solchen Gelegenheiten gesagt wird. Es ist nicht immer einfach, ihn zu verstehen, besser, es ist ausgesprochen schwer, ihm zu folgen und am Ende mischt sich alles in ein großes Klangbild. Das Publikum hat ihm mit einem sehr, sehr lang anhaltenden Applaus gedankt.» 
Schreibtisch das kleine Heft so selbstverständlich lag, war auf der anderen Seite das Erstaunen darüber groß, dass ich es eigentlich gar nicht besitzen sollte.

Es handelt sich also um einen Text, der direkt aus der >Werkstatt stammt und sich darin auch von Eggers Publikationen unterscheidet. Zu Eggers Arbeitsweise während dieses Jahres stehen im Jahresbericht wenige Hinweise: "Oswald Egger führte uns in seine neue Bibliothek ein, die er in Rom aufbaute. Nicht ein Tag über das Jahr, an dem Amazon ihm nicht Bücher lieferte. Nicht ein Tag. Ihm dann zuzuhören war ein Genuß, der uns nicht mehr verloren ging." (Blüher 2015, 17) Egger selbst beschrieb in unserem Austausch die Entstehung des Hefts so: Er wollte nicht, dass seine Bibliothek und seine Werkstatt auf Fotografien dokumentiert werden, er konnte die - mit einem Stipendium immer verbundene - >Leistungsschau für sich nur >durchbrechen`, indem er ızwar alles zeige, aber dafür nicht gerade lesbar macher. Ich hingegen habe mich dem Heft, so wie es vor mir liegt, angenähert und es folgt das Protokoll einer Lektüre - einer zufällig möglich gewordenen, aber eigentlich unmöglichen Lektüre. Es ist eine Lektüre, die auch die Entstehung einer Leserin-Position miterzählt.

»Lesen ist immer Jetzt. Freilich trag ich immer Bücher umher. Nicht beziehungsorientiertes Begehren. Ich könnte sie lesen, aber ich will nicht.« (Cotten 2019, 243) Diese Aussage, Ann Cottens Erzählsammlung Lyophilia (2019) entnommen, beschreibt mein Verhältnis zu den Büchern Oswald Eggers ziemlich genau. Während ich andere Bücher >verschlinge`, trage ich diejenigen von Egger mit mir herum, sie stapeln sich auf meinem Schreibtisch oder sie liegen aufgrund ihrer Größe und Schwere - so im Fall von Die ganze Zeit - neben meinem Bett. Das Begehren, das sich sonst im >Jetztı der Lektüre auflöst, bleibt hier in einer seltsamen Schwebe bestehen. Manchmal habe ich das Gefühl, dass mein Nicht-lesenWollen auch damit zu tun hat, dass die Bücher nicht gelesen werden wollen und dass sie extra Schwellen markieren und Mauern um sich herum aufbauen.

Dennoch schlage ich irgendwann eine Seite auf und lese ein bisschen, das beste Wort, das mir dafür einfällt, lautet: Ich ıschnäugge` in ihnen. Dies zeigt bereits: Das in diesem Beitrag verwendete >Ich < ist ein spezifisches, das in jeder Annäherung an den Text, in jeder Handlung und Assoziation, von der eigenen (Lese-)Biographie mitbestimmt ist. Es wird sich im Verlauf dieses Protokolls als eine Leserin-Position konturieren, die nur behelfsmäßig mit Stichworten wie ıschweizerisch`, >weiblich`, >akademisch`, von ıästhetischen und psychoanalytischen Theorien geprägt ‘ charakterisieren lässt. Laut dem Schweizerischen Idiotikon heißt »schnäugge«: Ich schnüffle, schnuppere, schnobere (nach Art der Hunde oder Schweine) darin; ich stecke meine Nase hinein (»vorwitzig, unerlaubt, heimlich, unordentlich«), ich stöbere darin herum, ich spähe aus und spioniere, was es dort zu sehen gibt (Schw. Idiotikon, Bd. IX, Sp. 1180-1182). 
`Schnäugge` kann ebenso eine oberflächliche Lektüre meinen, wie aus einer Schale mit Speisen nur das Beste herauszusuchen. Es umfasst die klammheimliche Neugierde (mit ihrer leichten Scheu) ebenso wie das unordentliche Herumwühlen im Schlamm. Es ist ein Wort, das viel mehr enthält, als ein einfacher Begriff sagen könnte, und deswegen passt es für mich so gut zu Eggers Texten.

Ich gehe also mit Eggers Texten auf eine Weise um, wie sie auch Christina Weiss beschreibt, die seine Bücher als »nicht gemacht für kontinuierliches, lineares Lesen« erachtet: »Es ist viel anregender, sie in unterschiedlichen Stimmungen aufzuschlagen und den Assoziationen zu folgen. Sie sollten immer auf dem Tisch liegen, man sollte sie unterwegs lesen, im Freien oder während man durch Stadt und Natur fährt. Diese Texte öffnen Spielräume, in die man Erinnerungen an Gelesenes, Geschehenes, Gehörtes, Erlebtes einbringen kann.« (Weiss 2007, 279) Weiss wiederum beschreibt hier etwas, das auch Oswald Egger in einem »Briefgespräch« mit Michael Braun als eine Art Hausgemeinschaft zwischen Buch und Leser*innen formuliert hat:

Aber als Hausbuch sollte sich indessen eigentlich eine gewisse Gewohnheit (Wohneinheit) einstellen; indem man irgendwo aufschlägt, eine Stelle oder Zeile liest, solange sie gefällt, oder Blätter überschlägt, Kapitel, und selber, vielleicht sehr bald schon, eigenen Gedanken unvordenklich folgt. ${ }^{4}$

Egger im Interview mit Braun [2000]

Egger imaginiert hier die Handlungen seiner Leser*innen - vielleicht auch mit seinen eigenen Lese-Gewohnheiten im Sinn, das Leben mit den täglich hoffentlich nicht nur von Amazon gelieferten Bücher. Mit dem Buch wohnen; wenn die Lust da ist, es mal aufschlagen und lesen, wobei das Gefallen die Dauer dieses Lesens bestimmt; blättern, umblättern und überblättern, sich also durch das Buch wie durch einen Raum hindurch bewegen, und zuletzt den eigenen Gedanken folgen. Die dann gedachten Gedanken sind sunvordenklich ‘, d. h., sie wurden vorher nicht gedacht und sie konnten nicht svorgedacht « werden. Der Text scheint nicht Gegenstand von Gedanken, sondern viel eher Anlass dafür zu sein, dass die Gedanken gedacht werden können. Die genannten Tätigkeiten umschreiben eine Bewegung von der durchaus auch körperlichen Hinwendung zum Buch zu einer gedanklichen Bewegung, die mich in meine Vorstellungswelt führt - und damit auch wieder aus dem Buch heraus.

Meinem Griff zum Idiotikon, dem Einbringen von eigenen Assoziationen von Weiss und Eggers >Wohneinheit Lesen zum Thema wird und die Handlungen, die um das seigentlicher Lesen herum passieren, zum Lesen dazu gehören.

4 Vgl. zum $>$ Hausbuch auch Vedder [2014]. 
Der vorliegende Band hat sich zum Ziel gesetzt, der Wörtlichkeit von Eggers Texten nachzugehen und nicht, wie bislang überwiegend geschehen, auf der Oberfläche ihrer lautlichen Eigenschaften entlangzugleiten. ${ }^{5}$ In Mikro-Lektüren soll gegenüber poststrukturalistischer Unverfänglichkeit die Frage nach dem hermeneutischen Verständnis noch einmal neu gestellt werden. ${ }^{6}$ Dies werde ich im Folgenden auch versuchen - aber so wie in meinem Griff zum Idiotikon Philologie und Sinnlichkeit eine enge Bindung eingehen, die den poetischen Verfahren zu entsprechen scheint, möchte ich auch jene gemeinschaftliche Lebensform von Text und Leser`in einbeziehen. Zu einem >Verständnis` von Eggers Texten tragen Wörterbücher und Methoden der Philologie bei, aber wenn sich >Verstehen`vollzieht, dann geschieht dies genauso an den Schwellen der Lektüre: An dem Vor- und Nachher, in den körperlichen und affektiven Empfindungen, in den Handlungen, zu denen mich seine Texte veranlassen, und in den Assoziationen, denen ich nachhänge. ${ }^{7}$ Ich beschreibe, wie sich Eggers Text in meine Wahrnehmungen, körperlichen Verhaltensweisen, Sprachhandlungen einschleicht - und also, wie er in mir, durch mich handelt. Es gilt daher auch, die Bewegung in den Text hinein und aus ihm heraus zu vollziehen.

\section{Schauen}

Das Heft Anch'io sono pittore, über das ich im Prolog bereits einiges gesagt habe, liegt geschlossen auf dem Schreibtisch vor mir. Es ist ein leichtes Hochformat $(24 \times$ $18 \mathrm{~cm}$ ), umfasst acht Bogen und ist mit einer Klammerheftung gebunden. Titel und Autorname stehen auf dem Cover in der Mitte in einem weißen Balken, während oben und unten Bilder zu sehen sind: Oben weiße Linien auf schwarzem Grund, unten schwarze Linien auf weißem Grund, jeweils mit einer rechten Hand, die in leicht unterschiedlicher Stellung einen Pinsel hält. Während die obere Zeichnung Assoziationen an eine Landschaft auslöst, bilden die unteren Linien eher ein abstraktes Netz. Später wird mir dazu eine Stelle aus dem Text auffallen: »Zumeist

5 So eine Formulierung von Martin Endres und Ralf Simon in einem Programmpaper für diesen Band (s. hier auch die Einleitung).

6 Mit Bezug bspw. auf Weiss 2007, De Felip 2013 sowie Albrecht 2017.

7 Vgl. ähnlich Weiss 2007, 280: »Dichter und Leser sind aufeinander angewiesen. Die Wortschöpfungen Eggers müssen in der Imagination des Lesers weitergeführt werden. Jeder wird das auf seine Weise tun. Egger findet ein schönes Bild dafür: das Fadenspiel, bei dem man aus der Hand des Partners eine Figuration übernimmt und sie verändert, bevor dieser sie erneut umformt." 
sinterne Marmoriermuster, Schnörkel-, Schlitz- und Sternorna- / ${ }^{8}$ mente, Zickzack-zuckende Kuckucks-Spiralen, meteorartige, Eilinien-rotierende Glutstreifen.« (Asp) Die Abbildungen auf dem Cover könnten neben Landschaften und Netzen auch von Adern durchzogene Marmor- oder Sternmuster zeigen.

Mit der Frage der Gegenständlichkeit und Abstraktion verbunden ist auch diejenige, ob die beiden Hände mit ihren jeweiligen Werkzeugen schreiben, zeichnen oder malen. Der Titel Anch'io sono pittore mag im Kontext des Stipendiums eine ironische Anspielung auf die Tradition der ausländischen Akademien in Rom - einflussreich war v. a. die französische Akademie in der Villa Medici - sein, die die zumeist klassizistisch ausgerichtete Ausbildung von Künstler*innen, das hieß lange hauptsächlich von Malern, fördern sollten. Gleichzeitig handelt es sich der Legende nach um einen Ausspruch, den Correggio getan haben soll, als er zum ersten Mal ein Bild von Raffael gesehen habe. ${ }^{9}$ Es ist ein doppeldeutiger Satz, der sowohl ein Initiationserlebnis beschreiben als auch einen Paragone intendieren kann. Seit Plinius’ Erzählung des Wettstreits zwischen den beiden Malern Apelles und Protogenes geht es dabei um die Frage, wer die schönere Linie malen kann (Mainberger 2007). Gleichzeitig zielt der Paragone stets auch auf das Verhältnis der Künste untereinander, ob der Malerei, der Bildhauerei, der Literatur oder der Architektur der Vorzug hinsichtlich der Möglichkeiten der Nachahmung zu geben sei.

Ich betrachte also die beiden malenden/schreibenden Hände und sehe gleichzeitig meine eigenen Hände, wie sie das Heft halten und den Umschlag umblättern, wodurch auf der ersten Seite der Text erscheint. Diese und alle folgenden Seiten sind bis an den Rand mit schwarzen Buchstaben und vereinzelten hellgrauen Zahlen (den Paragrafen) gefüllt. Mein Auge schweift über die Seite, ab und zu entziffere ich ein Wort (»Ruszel-Felsen«, »Einzelfließbilder«, Asp), manchmal hängt mein Auge und beginnt die Wörter aneinander zu fügen (»Es ist gewellt, gegriffelt, kühl«, Asp). Das Lesen bleibt aber sporadisch, es ist eher ein Anschauen dieser Fläche, auf welcher der Schwarz-Weiß-Kontrast von Papier und Buchstaben fast $\mathrm{zu}$ einem einheitlichen Grau verschmilzt. Das Lesen wird aber nicht nur durch diesen >Grauwert erschwert, ${ }^{10}$ sondern auch dadurch, dass die Zeilen am Ende einfach abbrechen, dass sie nicht - so meine Lesegewohnheit -

8 Die Querstriche bezeichnen jeweils die Stelle, bei der die Zeile aufhört und auf der nächsten Seite weitergeht.

9 Kolportiert bspw. in den vielgelesenen Historisch-kritischen Nachrichten von Italien von Johann Jacob Volkmann (1777, Bd. 1, 350).

10 `Grauwert ist ein Begriff aus der Typografie, der die Dichte des Satzes bezeichnet. Egger hat ihn beiläufig in unserem Gespräch erwähnt. 
auf der nächsten Zeile weitergeführt werden. Es ist, als ob mir der Text zu verstehen geben wollte, ich soll nicht lesen, sondern schauen. Ich nehme eine doppelte Schwelle wahr: Weil es Buchstaben und Wörter sind, will ich lesen und nicht schauen, aber weil das Lesen immer zum Abbruch kommt, höre ich wieder auf zu lesen und schaue. Der Zwischenraum von (Un-)Gegenständlichkeit, den bereits das Titelblatt aufgetan hat, tritt auch hier in Erscheinung und wirft das automatische Lesen auf sich selbst zurück. Ich beginne zu blättern und erkenne rasch, welche Handhabung erforderlich ist: Ich muss über den Seitenumbruch und dann über den Falz und wieder über die Seite hinweglesen - jede Zeile zieht sich vom Anfang bis zum Ende hindurch. Erst auf der allerletzten Seite angelangt, vollziehe ich mit dem Text den Zeilensprung, indem ich auf die erste Seite zurückspringe und auf der zweiten Zeile weiterlese.

Auch hier also werden Linien gemalt. Ich wähle irgendeine dieser Linien aus und werde wieder zum Kind, das lesen lernt, indem ich mit meinem Finger, in ständiger Berührung mit dem Papier, der Zeile entlangfahre. Am Ende angekommen muss ich sie festhalten, nehme einen zweiten Finger oder, besser noch, die zweite Hand dazu, damit mein Blick die Zeile auf der nächsten Seite nicht sogleich verliert. Statt um die Schönheit der gemalten Linie geht es hier um etwas anderes: Die Lessing'sche Unterscheidung der Literatur als einer Zeit- und der bildenden Kunst als einer Raumkunst wird an die Grenze der Aporie getrieben, wird die Linearität des Textes doch so sehr ausgereizt, dass der Text letztlich zur Fläche wird.

Während andere Texte von Egger durch ihre grafische Gestaltung eine Offenheit der Blick- und Leserichtungen entstehen lassen, die gegenüber einem slinearen dern, bin ich hier den unbeirrt und unbiegsam voranschreitenden - Prosa? Zeilen ausgeliefert. Ihr widerständiges Moment - einfach weiter zu laufen - realisiert einerseits die grundsätzliche Linearität der Literatur, richtet sich andererseits aber auch gegen das Lesen. Den Text >nicht gerade lesbar zu machen, wie es Egger in unserem Gespräch ausgedrückt hat, ist bereits für die materielle Anordnung grundlegend.

Der Text widersteht in seiner Materialität meiner Lektüre, die dadurch - paradoxerweise? - umso mehr eingefordert wird. Denn es ist - und dies sagt bereits einiges über Eggers poetische Verfahren aus - eine Unlesbarkeit mit System: Es sind 365 Paragrafen und ein Jahr lang war Egger Stipendiat in Rom, d. h., das Heft erschließt sich insofern als >Arbeitsjournal`, als es das Protokoll seines täglichen Schreibpensums darstellt, aus dem er dann später, so hat er es im Gespräch mit mir formuliert, seine Texte sherausdestilliert`. Gleichzeitig waren und sind Fragen der Zeit und ihrer Strukturierung stets zentral für Eggers Poetik, bereits dem Titel nach in Die ganze Zeit und auch in nihilum album, einem Band, der 
3650 Gedichte bzw. Lieder enthält, zehn also für jeden Tag des Jahres. Das letzte Wort von Anch'io sono pittore lautet »immer« (Asp) und bereits dadurch verweist der Text auf sein Interesse an der Ewigkeit und also an der Totalität der Zeit. Dabei handelt es sich nicht nur um ein sInteresse seiner Gestaltung nach selbst eine solche Totalität, einen Zyklus, der ein Jahr darstellt: Er sperrt sich nicht nur gegen die Lektüre, sondern auch gegen die Buchbzw. Heftform, in die er eingefügt wurde. Ich folge dem sich aus der schwierigen Hand-Habung ergebenden Impuls, von den Seiten Fotokopien herzustellen, sie aneinander $\mathrm{zu}$ reihen und $\mathrm{zu}$ kleben und so tatsächlich den fortlaufenden Text herzustellen, als den er sich ausgibt. Am Ende der langen Reihe angelangt, muss ich die letzte Seite an die erste kleben - Anfang und Ende miteinander verbinden - und immer an dieser Klebestelle macht mein Blick einen kleinen Knick und springt auf die folgende Zeile, setzt zur nächsten Runde an. Ich erhalte so - auch dies heißt, Eggers Texte >wörtlich` zu nehmen - einen geschlossenen Textkreis, setze mich in seine Mitte, drehe ihn um mich herum oder mich in ihm um meine eigene Achse, durch alle 365 Paragrafen hindurch. Im Text gibt es keine Lücken, keine Absätze und damit auch keine Pausen - ein >Tag، folgt auf den nächsten. Ein Jahr in einem Text - damit ist nicht nur ein gedanklicher Anspruch verbunden, der die Totalität im Kleinen behauptet. Es ist ein Anspruch, der mich in seiner materiellen Realisierung bereits etwas schwindeln lässt, bevor ich überhaupt begonnen habe, >richtig` zu lesen. Weil der Text so schwer >lesbar`ist, rückt seine Materialität und damit meine Annäherung an ihn, meine Hand-Habung, in den Vordergrund - er löst körperliche, und das sind auch: affektive, Reaktionen aus.

\section{Lesen}

Durch seine strenge Strukturierung sendet der Text also - Lesbarkeit hin oder her - deutliche Signale aus, doch gelesen werden zu können, vielleicht auch zu wollen. Ich beginne also mit der ersten Zeile bis zum Ende der ersten Seite:

1. Staub dampfte in dicken Wolken über die Kolosse und begrub kochende Ungestalten in seinem Mulm. Die Luft sott walmig atmend und lastete fast schwer. Risse

(Asp)

Staub, Wolke, Luft, dampfen, kochen, atmen - Kolosse, Ungestalten, Risse, begraben, lasten, schwer. Ich nehme in der ersten Lektüre ssemantische Wolken « der Gegenstand von Eggers poetologischer Metapher erscheint hier schon ganz am Anfang des Textes (Egger im Interview mit Weiss 2010) - wahr, die ineinander verschlungen, zwei verschiedene Konnotationen haben: Leicht und schwer, flüchtig und materiell, aufsteigend und herabfallend, entstehend und verge- 
hend. Die Substantive sind durch Verben im Präteritum miteinander verbunden: ein episches Präteritum, ein Blick aus der zeitlichen und räumlichen Distanz, ohne erkennbare Aussageinstanz. Ich bin in eine Landschaft geworfen, es stellt sich ein zunächst durchaus kohärentes Vorstellungsbild vor meinem inneren Auge ein: Wasserstaub zu Wolken verdichtet, die um und über kolossalen Bergformationen schweben - eine Atmosphäre, in der sich Luft so sehr verdichtet, dass sie materiell wird, physische Präsenz erhält, ohne eine Gestalt anzunehmen. Erinnerungen an Wanderungen stellen sich ein. Gleichzeitig assoziiere ich literarische und mythologische Vorstellungen: Die Genesis, der Geist Gottes über einer uranfänglichen Welt schwebend, Materie am Übergang von Nichts zur Gegenständlichkeit, Werden und Vergehen als ewiger Zyklus: »Denn Staub bist du / zum Staub musst du zurück." (Gen. 3, 19) Und ich merke, wie ich zunächst das Wort `Koloss` metaphorisch für Berge gelesen habe, erinnere mich an seine Bedeutung >Riese Ton des epischen Präteritums - vor allem diese Bedeutung, die in mir auch das Bild des riesenhaften Titanen Atlas hervorruft, der, den Uranos stemmend, das Zusammenfallen von Himmel und Erde verhindert und so das Thema mythologischer Urgeschichte(n) der Erde anklingen lässt. Im Zusammenhang mit Rom denke ich zudem unweigerlich an die Kolossalstatue Konstantins des Großen, deren Fragmente in den Kapitolinischen Museen ausgestellt sind. Zwischen Wanderungen und Kolossalstatue zeigen sich erneut - sowohl private wie auch berufliche - biographische Aspekte dieser Leserin-Position, und es wird deutlich, wie offen der Text für diese ist, aufgrund seiner großen Zurückhaltung gegenüber spezifisch raum-zeitlicher und semantischer Konkretion.

Auch wenn Egger die Analogie zwischen der Genesis und einer >dichterischen Schöpfung، ablehnt (Egger im Interview mit Braun [2000]), so komme ich veranlasst durch die oben beschriebene Kreisform des Textes - auch nicht umhin, die semantische Ebene mit dem Zyklus eines Jahres als Zyklus der Natur und des Lebens zu verknüpfen. Ich erinnere mich an die Aussage, wonach »der ganze Kreis der Erde [...] gleichsam in der Rede wiederkehren « müsse, ${ }^{11}$ wobei das Anagramm (Rede/Erde) tatsächlich in der potenziellen Kreisform von Anch'io sono pittore realisiert ist.

Der zweite Teil des ersten Satzes (»und begrub kochende Ungestalten in seinem Mulm«) lässt sodann meine Vorstellungen innehalten und verändert sie. Die Leerstellen des Sinns öffnen weitere Lücken - Egger hat es einmal als saugenblitzende Luken` bezeichnet (Egger im Interview mit Braun [2000]) -, in die

11 Egger im Interview mit Braun [2000]. Das Zitat findet sich in Egger 2003, 42. Vgl. auch Endres 2014. 
meine Imagination hineinschlüpfen kann. Ich versuche assoziierend zu paraphrasieren: Der Staub begräbt - d. h., er ändert seinen Aggregatszustand, er ist nicht mehr metaphorischer >Wasserstaub<, sondern doch eher Staub aus Erde, der auf etwas herabstürzt. `Etwas`, d. h. hier: »kochende Ungestalten « - und wiederum verkehrt sich der Aggregatszustand bzw. meine Vorstellung. Zwar kann ich die >Ungestalten ‘ auf die `Kolosse` beziehen - als `plumpe, hässliche Gestalten ${ }^{12}$ können sowohl die Felsformationen als auch die Riesen bezeichnet werden. Aber so wie das Wort `Gestalt` im Wort `Ungestalt` negiert wird, wird hier sogar noch die Ungestalt dieser Gestalten aufgelöst in etwas 'Kochendes`. Der Wasserstaub wird zu Erde, die Felsen werden zu Wasser. Vom ersten zum zweiten Satzteil, die durch das sund - die Konjunktion der Gleichzeitigkeit - miteinander verbunden sind, findet eine doppelte Inversion statt. Zur Kreisform auf der materiellen Ebene und zum Thema des Werdens und Vergehens der Genesis auf der thematischen Ebene kommt hier eine dritte zirkuläre Bewegung in den Blick und realisiert sich auf der syntaktisch-semantischen Ebene des Wortmaterials. In meinem Versuch, dem Text Wort für Wort zu folgen, tut sich eine Differenz auf zwischen dem Begreifen- und Beschreiben-Wollen (die mich schwindeln lässt, weil ich die Orientierung verliere) und dem Gefühl (das doch irgendwie stimmig ist, weil genau das passiert, was ich zu beschreiben versuche). Was hier aus der Aneinanderreihung von Buchstaben und Worten entsteht, ist nicht die Totalität der Welt, aber eine totale Rede, die in alle möglichen Richtungen geht und Assoziation über Assoziation schichtet - mythologische Vorstellungen eingeschlossen.

Dabei ist der Satz ja noch gar nicht zu Ende: Der Staub begräbt die Ungestalten »in seinem Mulm« - und ja, genau, `mulmig`, so hätte ich das eben formulierte Gefühl auch bezeichnen können. Der Satz agiert nicht allein, indem er mich in einen bestimmten affektiven Zustand versetzt - er spricht, freilich indirekt, diesen Zustand auch aus, benennt ihn mit einem Wort, das ich nur halb verstehe, aber gerade deshalb irgendwie mit mir in Verbindung bringen muss, um überhaupt etwas mit ihm anfangen zu können: Indem ich den »Mulm« mit meinem Gefühl bei der Lektüre identifiziere, werde ich quasi zu dem, was der Staub hier >begräbt.$^{13}$ Aus dem Grimm'schen Wörterbuch, zu dem ich dann doch greife, erfahre ich, dass >mulmig` tatsächlich von >Mulm` abgeleitet ist, das seinerseits "zerfallene erde, staub" bedeutet und aus "mahlen, zerreiben" gebildet wurde. »Mulm« bezeichnet das zu Erde zerfallene Holz (»dahero auch, wann von selbem (baume, der rotbuche) ein ast gehauen, in wenig jahren sol-

12 So der Artikel »Ungestalt« im Duden (Dudenredaktion).

13 Damit ist eine umstrittene, aber dem Lesen oft zugesprochene Leistung thematisiert, die nicht erst die Neurowissenschaft anhand der Theory of Mind und den sog. Spiegelneuronen zu erklären versucht. Vgl. bspw. Rosa 2020. 
cher hieb müllmicht wird, dasz die spechte den mulm heraus hacken und ihre jungen darinnen aufbringen. Hohberg 3, 2, 330a«) und auch »sonst wie erde zerfallendes« (»die erdäpfel sind zu mulm verkocht. Göthe 11, 94«) (DWB, Bd. 12, Sp. 2657 f.). Die zirkuläre Struktur wiederholt sich noch einmal, macht einen Dreh weiter bis zur Tautologie - denn verkürzt lautet die Paraphrase dieses Satzes nun: Der Staub begräbt unförmige Gestalten in seinem Staub. Das Wörterbuch klärt zwar die Bedeutung des Wortes, aber zugleich ist auf zweifache Weise keine neue >Information، zu diesem Satz hinzugekommen: Einerseits, weil Staub eigentlich ein Synonym von Mulm ist - und ich diese semantische Ebene also bereits so erfasst hatte - und andererseits, weil ich durch meine Assoziation mit meinem mulmigen Gefühl bereits irgendwie verstanden hatte, was das Wort heißt, bevor ich seine tatsächliche Bedeutung erfasst hatte.

Aber im Nachvollzug der Tautologie, die die unsinnige Paraphrase offenbart, ist doch etwas passiert. Der Text hat die Drehung in sich selbst, worauf er bereits in seiner Materialität der Kreisform hingewiesen hatte, noch einmal performativ wiederholt. Er hat auch das Thema der Weltentstehung weiter verdichtet und poetologisch als Frage der Text-Entstehung ausgestellt. An diese inständige Selbstreferenzialität ließen sich poststrukturalistische Lektüren anschließen, die - wie es etwa Eleonore de Felip versucht hat - den ständigen Aufschub von Sinn reflektieren: »Es ist ein Gehen durch offene, mentale Räume, durch sinwendige Landschaften, das Lesen ist ein Sammeln und Blüten-Lesen in Wortgärten und ein Verweilen in den Leerräumen der Sprache.« (De Felip 2013, 70) Doch ein solcher Zugang hat seine Grenzen bereits, wenn auch nicht allein, darin, dass sich die Resultate entsprechender Lektüren in den letzten Jahren immer ähnlicher geworden sind - ganz egal, welchen Text sie zum Gegenstand haben. Vernachlässigt wird zudem, dass die tautologischen Strukturen insofern affirmativ sind, als sie über eine Handlungsdimension verfügen, die mich als Leserin direkt betreffen. Indem mir in der Kreisbewegung zwischen dem ersten und dem zweiten Satzteil schwindlig-mulmig geworden ist, findet eine Übertragung von den sbeschriebenen Gegenständen`, seien sie poetologisch zu verstehen oder nicht, auf mich statt und ich begebe mich selbst in die Kreisbewegung hinein. Die Haltlosigkeit dieses Lesens findet in lautlichen und semantischen Assoziationen und in meinen affektiven Zuständen Ankerpunkte - bevor ich das Wort `Mulm` nachschlage, hatte ich bereits eine Ahnung davon, was damit gemeint ist und im Nicht-Verstehen macht das Wort etwas mit mir. Wiederum hat der Text mich auf mich selbst zurückgeworfen, aber er hat mir auch gezeigt, dass es ein Verstehen im Nicht-Verstehen gibt. So etwas in der Art formuliert auch Egger - unklar, ob er dabei seine Leserin oder sich selbst im Blick hat -, wenn er in dem bereits zitierten Interview die Empfindung beim Lesen «mit Augen und Herz« betont: »Wortaggregate müssen einen ansprechen, damit man sie aufliest. Man wird Wort für Wort annehmen und diese 
Annahmen mit Augen und Herz lesen. Irgendwie. Wer es als störend empfindet, dass er nichts wirklich wieder erkennt, wird den Text fortlegen.« (Egger im Interview mit Weiss 2010)

Ich lege den Text noch nicht weg, sondern lese noch den zweiten Satz der ersten Zeile (»Die Luft sott walmig atmend und lastete fast schwer.» Asp). Der Wechsel der Aggregatszustände wird ebenso weitergeführt, wie die Wortreihen `Staub Wolke - Luft « sowie >dampfen - kochen - sieden` fortgesetzt werden. Die Assoziationen an die Genesis werde ich nicht los, ist es doch der >Atem` Gottes, der über den Wassern schwebt (Gen. 1, 2). Und auch die Verdrehungen werden fortgesetzt die Luft siedet nicht nur, sie lastet auch (fast) schwer. Die Vorstellungen des ersten Satzes werden so zusätzlich angereichert und gesättigt.

Hier lässt sich zudem zeigen, wie sich das Verstehen im Nicht-Verstehen von Wort zu Wort angelt. Bei dem mir unbekannten Wort »walmig « fühle ich mich in meiner Bildung von >mulmig` aus `Mulm` bestätigt und übertrage das damit verbundene Gefühl auch auf dieses Wort, wobei sich zusammen mit >Atmen sschwer die Assoziation zu >wallen` einstellt. Unter den Eintragungen im Wörterbuch zum Substantiv »Walm» finden sich die Bedeutungen 1) »bewegung des Wassers, wasserschwall, strudel« 2) »aufwallen heiszen wassers, sieden«, auch im übertragenen Sinn (Gemüt aufwallen) (DWB, Bd. 27, Sp. 1316-1318). Indem ich das Wort also wiederum verstanden und nicht verstanden, d. h. nicht begriffen, aber im Verbund mit den anderen ein Gefühl dafür entwickelt habe, wird wiederum ein affektiver Zustand evoziert, der die - durchwegs anthropomorphisierten - Gegenstände im Text, das Wasser, die Luft, ebenso betrifft wie mich selbst: Mir ist mulmig, ich komme ins Wallen, gerate in Wallung, ich atme schwer. Nicht Gottes Atem, sondern mein Atem schwebt über dieser Textwelt.

Ob Zufall oder nicht - das letzte Wort dieser ersten Zeile lautet »Risse« und benennt wiederum das, was beim Umblättern der Seite passiert. Ich lese aber weiter, diesmal über die ganze Doppelseite und noch ein bisschen weiter:

und dazwischengepferchte Schritte, die erstickten. Wie wenn Hirse blüht zerrupftere Garben im Karst: Die Tiefe schnatterte und schob sich, paffte und roch oder tra / mpelte schon, als lochförmig blitzzischende Striche empor aus der tobeligen Tiefe, Klamm-wärts, die Felsknollen rogelten von Schwefelgrannen abschellernde Metall / -Klexe wie dann die Wut den Lärm begrub:

(Asp)

Das Menschliche, das ich in meiner Lektüre, auf mich selbst Bezug nehmend, schon antizipiert habe, kommt nun tatsächlich in den Text hinein, in Form von Schritten, die ersticken. Das Ersticken schließt dabei nahtlos an das mulmige Gefühl, das schwere Lasten und Atmen an. Auch meine Versuche, den Text zu durchschreiten, ersticken immer wieder. Wie sich Vorstellungsbilder andeuten, aber in den Wendungen und Anreicherungen des Textes sich wieder auflösen, 
wird am folgenden Satz deutlich: Das Stilmittel des Vergleichs (১wie wenn ) bewirkt, dass sich meine Vorstellung von etwas erweitert, indem etwas `Eigentliches` mit etwas >Uneigentlichem` verbunden wird. Hier werden nun die ungestalten Felsformationen konkret als eine Karstlandschaft benannt, deren Spalten wie szerrupfte، Garben von blühender Hirse - welche Hirsenart, müsste man fragen - aussehen, wobei zuerst das Bild und dann der zu beschreibende Gegenstand genannt wird, was das Verhältnis von Vor- und Nachbild bereits problematisiert. Mich an meine einige Wochen zurückliegende Wanderung durch ein Karstfeld erinnernd, fällt es mir zudem zunächst schwer, diese kargen Felsen, wo fast nichts wächst, plötzlich mit blühendem Getreide zu verknüpfen - und auch im Text steht dieses Organische in Kontrast zu den Elementen von Luft, Wasser und Fels. Einerseits findet durch die Nennung des geologischen bzw. biologischen Namens von Karst und Hirse eine plötzliche und überraschende Konkretisierung statt, aber die Schwierigkeit besteht darin, meine Erinnerungsbilder von Karst/Hirse miteinander zu verknüpfen. Erst mit der Zeit gelingt es mir und ich kann die Risse zwischen den Felsen, die Negativformen als Garben der Pflanze betrachten (und gleichzeitig macht mein Auge aus `Garben` immer wieder >Graben` und passt damit das Wort meinen bekannteren inneren Karst-Bildern an). Dabei passt der Vergleich über diese Kluft hinweg wiederum zum leicht surrealen - zwischen Traum und Wirklichkeit stehenden - Gefühl, das ich bei meiner letzten Durchwanderung dieser ungestalten Felsen hatte, die mich - wie die Wolken - dazu verführen, mir in ihren Gestalten Gegenstände, Tiere oder Gesichter vorzustellen. ${ }^{14}$

Der erste Teil des folgenden Satzes hat erneut eine zirkulär-tautologische Struktur - ist es doch die Tiefe, die aus der Tiefe empor schnattert, sich schiebt, pafft, riecht und trampelt. Sie ist einerseits der Ort, wo diese Bewegungen und Sinneswahrnehmungen herkommen, und sie wird zugleich belebt. Doch die genannten `Handlungen`, die sie ausführt, sind wahlweise mit einem `und >oder verbunden und erscheinen so - was in einem Widerspruch zum epischen Präteritum steht, von dem ich eine Schilderung der tatsächlichen Geschehnisse der Vergangenheit erwarte - als verschiedene Optionen: Das Terrain meiner Vorstellung, das bereits beim Vergleich Karst/Hirse lückenhaft war, bleibt unsicher. Die Bilder verdichten sich nicht zu einer geschlossenen Vorstellung, die Risse bleiben. Es ist klar, dass es nicht um die Beschreibung einer Landschaft geht, sondern um Möglichkeitsbilder und die Möglichkeiten meiner Vorstellungen, aber gleichwohl bleibt der Text auf eine gewisse Verankerung in der evozierten >Naturlandschaft ‘ angewiesen. Die Möglichkeitsbilder werden in mir zu

14 Vgl. da Vinci 1882, Par. 66. Sowie aus der Forschungsliteratur: Rosenberg 2004 sowie Wagner 2006. 
einer Wirklichkeit, solange ich nicht versuche, sie festzuhalten, sondern mich von ihnen - ein gewisses Tempo spielt hier durchaus eine Rolle - vorwärtstragen lasse.

Zentral dafür ist der Klang; es zeigt sich immer mehr, dass die klanglichen Äquivalenzen konstitutiv für das Erfassen des Textes sind: Etwa durch die Häufung

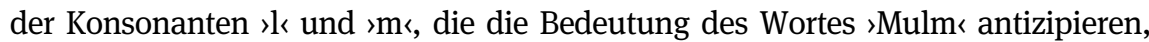
bevor ich es verstehe, ${ }^{15}$ oder der durch die sich fast regelmäßig abwechselnden runden, dunklen Vokale sa und >o< gebildete Untergrund, der dann plötzlich von scharfen ıZisch-- und ii-Lauten durchbrochen wird. ${ }^{16}$ Wie ich mit dem Finger den Buchstaben entlangfahre, die Zeile nachzeichne, um mich nicht im Text zu verlieren, so komme ich auch vor allem mit meinem Gemurmel durch den Text. Alles andere als in einem stillen Lesen vollziehe ich den Text klanglich nach, Finger und Mund sind in der Handhabung eng aneinandergebunden.

Ich bin noch nicht einmal am Ende des kurzen, oben zitierten Abschnitts angelangt und doch ist das Geflecht an Bezügen und Assoziationen bereits so vielgestaltig, dass ich Mühe habe, allen Verästelungen dieses doch eigentlich auf einer einzigen Linie voranschreitenden Textes nachzugehen und all das, was sich dabei gewissermaßen als Raum auftut, in eine Paraphrase zu übersetzen. Mein Auge hängt an dem Wort »Metall-Klexe«, das über die zwei Seiten aufgespannt ist, vielleicht weil mir hier zuerst auffällt, dass unter den Formen

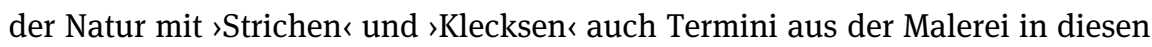
Text hineinkommen und damit direkt auf den Titel Anch'io sono pittore zurückweisen. Der Bereich zwischen Gegenständlichkeit und Ungegenständlichkeit, den ich oben in Bezug auf das Cover beschrieben habe, wird nun auch in Bezug auf den Text konkreter fassbar: Aus dem Lautmaterial und meinen Assoziationen kristallisieren sich während der Lektüre immer wieder Ansätze von Vorstellungen heraus, worauf im nächsten Moment wieder die Lautstruktur das Bild in Bewegung versetzt und zur Auflösung bringt - es ist ein, so hat es Paul Valéry formuliert, »Zaudern zwischen Laut und Bedeutung ${ }^{17}{ }^{17}$ Die sich kreuzenden Linien auf dem Cover, aus denen Landschaften, Marmormuster und Sternornamente hervorgehen, gleichen so den Verknüpfungen, die ich aus diesen Buchstabenreihen irgendwo zwischen meinem lesenden Finger, meinem murmelnden Mund und den Bildern in meinem Kopf herstelle. Dass in diesem Text »Marmoriermuster, Schnör-

15 In der Wortreihe »kochende Ungestalten in seinem Mulm. Die Luft sott walmig atmend und « (Asp).

16 Vgl.: »Die Tiefe schnatterte und schob sich, paffte und roch oder trampelte schon, als lochförmig blitzzischende Striche empor aus der tobeligen Tiefe« (Asp).

17 Roman Jakobson $(1979,106)$ zitiert diese Formulierung in seinem Beitrag Linguistik und Poetik. 
kel-, Schlitz- und Sternorna- / mente, Zickzack-zuckende Kuckucks-Spiralen, meteorartige, Eilinien-rotierende Glutstreifen« (Asp) vorkommen und »daß Striche, Vertiefungen und Höfe mit Ungründen zeigen, am Bod / en, die zu einer vorgefaßten Figur unzusammengestanzt tanzen « (Asp), ${ }^{18}$ erinnert mich daran, wie Walter Benjamin in seinen Überlegungen Über das mimetische Vermögen und Über die Lehre vom Ähnlichen das Lesen dargestellt hat:

»Was nie geschrieben wurde, lesen.« Dies Lesen ist das älteste: das Lesen vor aller Sprache, aus den Eingeweiden, den Sternen oder Tänzen. Später kamen Vermittlungsglieder eines neuen Lesens, Runen und Hieroglyphen in Gebrauch.

(Benjamin 1991, Bd. II.1, 213)

Die Linien und Muster von Eingeweiden, Sternen und Tänzen werden zu Anlässen erster mimetischer Handlungen, aus denen auch die Sprache hervorgeht. Es ist ein uranfängliches Lesen, bei dem es gegenüber der zwar arbiträren und konventionellen, aber eindeutigen Bezeichnung vielmehr um den Vorgang, Ähnlichkeit herzustellen, geht. Egger nimmt in dem bereits erwähnten Briefgespräch wohl auf dieses >Denkbild〈 Benjamins Bezug: »Zu den wichtigsten Denkbildern gehört für mich das Bild von der Sprache als salle Sterne`. Jeder kann sich seine eigenen Sternbilder isolieren. Wort-für-Wort-Konstellationen - das sind Sternbilder, aber man muss sie sich erst suchen und ausdenken." (Egger im Interview mit Weiss 2010) Indem ich $\mathrm{zu}$ einem mit dem Finger und stammelndem Mund lesenden Kind werde, lese ich mir aus den Lauten und Worten einen Text zusammen. Die Sterne sind da und ich stelle Verbindungen her: Anch'io sono pittrice!

\section{Hinlegen}

Ich bin dem Text ein Stück weit gefolgt, aber nicht über die ersten wenigen Sätze hinausgekommen. Mir schwirrt der Kopf, was für die philologisch-hermeneutische Arbeit nicht ungewöhnlich, aber hier doch noch einmal anders ist. $\mathrm{Zu}$ den Handlungen, zu denen mich Eggers Texte veranlassen, gehört, wie auch jetzt, mich immer wieder hinzulegen und den Blick vom Heft wegzuwenden. Sind die Schwellen - auch diejenigen der Handhabung - einmal überwunden,

18 Das ganze Zitat aus den Par. 22./23. lautet: »Zumeist sinterne Marmoriermuster, Schnörkel-, Schlitz- und Sternorna- / mente, Zickzack-zuckende Kuckucks-Spiralen, meteorartige, Eilinienrotierende Glutstreifen. 23. Am besten gelingen mir die Experimente selbst mit meinen Händ / en, ganz wunderliche Augen-Gestalten. Ich glaubte, sie alle nacheinander abzeichnen zu müssen, daß Striche, Vertiefungen und Höfe mit Ungründen zeigen, am Bod / en, die zu einer vorgefaßten Figur unzusammengestanzt tanzen.« (Asp) 
so hat sich gezeigt, dass ich dem Text durchaus auf eine Weise folgen kann, dass ich mehr verstehe, als ich zunächst gedacht habe. Ich bin auch deswegen so randvoll mit diesen wenigen gelesenen Worten, weil ich die Bewegung in den Text hinein dadurch gefunden habe, indem ich mich ihm ähnlich gemacht habe - weil er meinen Finger, meinen Mund, meine Vorstellungen involviert und weil diese >Landschaft immer mit der inneren >Landschaft ' meiner Affekte interagiert hat.

Die Anstrengung - und eine Anstrengung bleibt es auch dort, wo sich plötzlich eine sLuke، auftut - besteht darin, dass ich mit jedem Wort einen neuen $\mathrm{Zu}$ gang suchen muss. Sie hat entsprechend auch zur Folge, dass ich immer wieder ganz hinausfalle. Wenn ich auf dem Sofa liege, dann bin ich nicht - wie bei anderen literarischen Texten - weiterhin in der Geschichte drin, mit den Protagonist*innen zusammen oder halte mich in den Landschaften und Räumen auf. Sobald ich den Text weglege, ist er nicht nur physisch weg, es bleibt auch wenig konkret Erinnertes da. Es ist vielmehr eine Bewegung vereinzelter Vorstellungsfragmente - Wolken, Staub, Felsformationen, Karstlandschaften, Schluchten und Pflanzen, Striche und Kleckse, die ich beobachten kann - und da ist dieses vage Kribbeln.

Ich fühle mich wie eine jener Glaskugeln, die man durchschüttelt, damit der Schnee in ihnen herumwirbelt. Vielleicht fällt mir dieses Bild ein, weil ich schon vorher bei Walter Benjamin war, der - ein passionierter Sammler solcher Schneekugeln ${ }^{19}$ - das Lesen eines Kindes mit dem Betrachten von Schneeflocken verglichen hat, so bspw. im kurzen Text Schmöker, der 1933 unter dem Pseudonym Detlef Holz in der Vossischen Zeitung erschienen ist:

Beim Lesen hielt ich mir die Ohren zu. So lautlos hatte ich doch schon einmal erzählen hören? Den Vater freilich nicht. Manchmal jedoch, im Winter, wenn ich in der warmen Stube am Fenster stand, erzählte das Schneegestöber draußen mir so lautlos. Was es erzählte, hatte ich zwar nie genau erfassen können, denn zu dicht und unablässig drängte zwischen dem Altbekannten Neues sich heran. Kaum hatte ich mich einer Flockenschar inniger angeschlossen, erkannte ich, daß sie mich einer anderen hatte überlassen müssen, die plötzlich in sie eingedrungen war.

(Benjamin 1991, Bd. IV.1, 275)

Dem ununterbrochenen Schneien von Eggers Text kann ich mich teilweise anschließen, wobei ich nie alles in dem dichten Gedränge erfassen kann. Ich folge einer >Flockenschar`, nur um zu bemerken, dass sie sich bereits wieder verän-

19 Vgl. Theodor W. Adorno $(1970,14)$ über Benjamin: »Ihn sprachen die versteinerten, erfrorenen oder obsoleten Bestandstücke der Kultur, alles an ihr, was der anheimelnden Lebendigkeit sich entäußerte, so an, wie den Sammler das Petrefakt oder die Pflanze im Herbarium. Kleine Glaskugeln, die eine Landschaft enthalten, auf die es schneit, wenn man sie schüttelt, zählten zu seinen Lieblingsutensilien." 
dert hat. Dabei werden die Grenzen zwischen mir und dem Text zunehmend verwischt - der Erzähler in Benjamins Text beschreibt, wie »die Ferne, wenn es schneit, nicht mehr ins Weite, sondern ins Innere führt« und also all die fernen Länder, »Babylon und Bagdad, Akko und Alaska, Tromsö und Transvaal«, plötzlich in seinem Inneren lagen. Die »linde Schmökerluft«, die dem jungen Leser aus den Büchern entgegenwehte, »schmeichelte« sich dabei »unwiderstehlich [s]einem Herzen« ein. Das Rieseln des Schnees wie der Worte dringt ebenso unmerklich wie kraftvoll bis auf die Knochen ein (Benjamin 1991, Bd. IV.1, 275). Fünf Jahre zuvor, im Kapitel »Lesendes Kind « der Einbahnstraße, beschreibt Benjamin diesen Prozess noch dezidierter als körperlichen Vorgang: Man geht in einen Text wie in ein Schneetreiben hinein, das einen ganz umfängt, und man ist für eine Woche "gänzlich dem Treiben des Textes anheimgegeben « (Benjamin 1991, Bd. IV.1, 113). Das Kind setzt sich noch mehr als Erwachsene diesem Geschehen aus: »[E]ine Hand liegt immer auf dem Blatt«, es hat ständigen physischen Kontakt zum Buch und so »sind [für es] die Abenteuer des Helden noch im Wirbel der Lettern zu lesen wie Figur und Botschaft im Treiben der Flocken«. Es geht in diesen Wirbel hinein »und wenn es aufsteht, ist es über und über beschneit vom Gelesenen. «(Benjamin 1991, Bd. IV.1, 113) ${ }^{20}$ Indem ich mit den Fingern den Zeilen nachfahre und die Klänge nachmurmle, indem ich körperlich involviert bin und die von den Worten evozierten affektiven Zustände auf mich beziehe, werde ich auch $\mathrm{zu}$ jenem Kind, das von den Worten des Textes durchschneit wird. Einen solchen Vorgang der Verwandlung eines >Ichs« benennt der Text auch wiederum selbst. So heißt es im 24. Paragrafen:

Das Wortlose windstiller Ahnun / gen und Annahmen, das Wegsinken oder Auftauchen von Meergegenden, einer Streuwiese, einem Dorfanger, Garten, einer Scheune, einem Gehöft mit geöffneten Fen / stern, ein Pinienhain ... das war ich alles selbst.

(Asp)

Erneut formuliert der Text, wie es mir bei seiner Lektüre ergeht - indem ich das Meer, die Wiese, die Pinien in mir entstehen lasse, werde ich ein Stück weit selbst $\mathrm{zu}$ ihnen. Es entstehen >wortlose Ahnungen`, die nicht an einen Begriff geknüpft, sondern mit dem Auftauchen und Verschwinden von Vorstellungsbildern verbunden sind. Es ist ein >Ich in Bewegung wie Benjamins Flockenschar, ohne feste Gestalt, ohne innere Kohärenz.

Eine Antwort auf Eggers Frage - »Was wird ein Wort schon sein, wenn ich aufhörte, es zu verschwenden an die Verständigung? «(Egger 2015b, 120) - könnte in diesem Sinne lauten: Der Text schenkt mir die längst vergessene Erfahrung, mich zu verwandeln, mich ganz - bereits Benjamin benutzt die von Egger verwen-

20 Vgl. hierzu Brüggemann 2007, 280-297. 
dete poetologische Metapher - »in die Worte, die eigentlich Wolken waren, mich zu mummen « (Benjamin 1991, Bd. IV.1, 261). Vielleicht schenkt er mir auch eine Erfahrung, die noch weiter zurückliegt und die - ich trage ein weiteres Element meiner Lesebiographie hier hinein - Didier Anzieu in Das Haut-Ich als Erfahrung des Kleinkindes einer »Lauthülle« beschrieben hat: Ich begebe mich in ein - wie das ursprünglich von der Stimme meiner Mutter gebildete - Wort- und Klang-Bad hinein und es wird eine Hülle bzw. Haut gebildet, eine Membran, an der mein Brabbeln und Stammeln, meine Affekte und Assoziationen und dann die Symbolisierungen entstehen (Anzieu 1991, 207-226).

Dieses regressive Moment wird erzeugt und bestärkt durch die Kreisform des Textes, mein Gefühl, mich unablässig um meine eigene Achse drehen zu müssen, von jeder Silbe und jedem Wort vorangetrieben $\mathrm{zu}$ werden, weil es keinen Halt gibt. Indem der Text sich bei mir einschmeichelt - das Ich zu allem und alles zum Ich wird -, nimmt er von mir Besitz und reißt alle Differenzen ein. Das Mulmige und der Schwindel sind Zustände der Beseßenheit, wie sie der Text selbst benennt, wenn das erste explizit affektive Wort die »Wut« ist, die in der obigen Passage »den Lärm begrub«. >Wut` ist, wieder laut Grimm, eine »heftige seelisch-leibliche erregung und ihre äuszerungen«, etymologisch verwandt mit »blasen, inspirieren « und »weissager, seher« und auch mit »ton, schrei, stimme, gesang, dichtung, beredsamkeit«. Vom rasenden Wahnsinnsanfall, der religiösen Schwärmerei oder der weltentrückten Anteilnahme des Dichters am Göttlichen ist damit stets ein Kontrollverlust verbunden (DWB, Bd. 30, Sp. 2474-2491). Ich gerate in die Wut des Textes hinein - aber wenn ich daraus aufwache, ist nichts mehr da. Sobald ich mich vom Text entferne, also, wie eben hier und jetzt, einfach daliege, so ist der Spuk vorbei und nur dieses vage Gefühl als Reminiszenz des (Schnee-)Treibens des Textes bleibt zurück. Es gibt keinen mittleren Zustand den Texten Eggers gegenüber. Es gibt nur drinnen oder draußen, totale Präsenz oder totale Absenz.

\section{Weiterlesen}

Ich setze mich wieder an den Schreibtisch, sschnäugge ein paar Brocken im Text. An einigen Stellen fällt mir der veränderte Duktus auf. Eine Recherche auf Google-Books (wie hat man früher bloß Wissenschaft betrieben?, frage ich mich einmal mehr) spuckt mir Überschneidungen mit zwei Abhandlungen von Siegfried Passarge aus: Wissenschaftliche Ergebnisse einer Reise im Gebiet des Orinoco, Caura und Cuchivero im Jahre 1901-02, im Jahr 1933 als Bd. 39 der Abhandlungen aus dem Gebiet der Auslandskunde erschienen. Und Morphologische Studien in der Wüste von Assuan, im Jahr 1955 (jedoch auf Untersuchungen aus dem Jahr 1914 
zurückgehend) als Bd. 60 in der gleichen Reihe publiziert. Siegfried Passarge (1866-1958) war Geograph, Geologe und Paläontologe und gilt als Begründer der Landschaftsgeographie. Obwohl er bereits in den 1920er Jahren mit seinen antisemitischen Äußerungen auffiel, wurde er 1925 Mitglied und 1926 korrespondierendes Mitglied der Bayerischen Akademie der Wissenschaften. 1929 publizierte er ein Buch mit dem Titel Das Judentum als landschaftskundlich-ethnologisches Problem. 1933 erfolgten trotz der Aufnahmesperre der Eintritt in die NSDAP und die Unterzeichnung des Bekenntnisses der deutschen Professoren zu Adolf Hitler. Die Publikationsreihe Abhandlungen aus dem Gebiete der Auslandskunde ist die Fortsetzung der Abhandlungen des Hamburgischen Kolonialinstituts, wo Passarge von 1908 bis 1936 Professor war. Ein Nachruf des Geographen Herbert Louis für die Bayerische Akademie hebt die wissenschaftliche Bedeutung von Passarge hervor, lässt aber keinen Zweifel daran, dass Passarge »[s]ehr entschiedene Vorschläge zur Kolonialpolitik [gemacht habe], in denen seine später immer erneut aufgegriffenen Gedanken zur Rassenfrage bereits anklingen«, und dass er glaubte, »Charaktertypen von Völkern unterscheiden und ableiten zu können «. ${ }^{21}$

Ich beginne, verschiedene Abschnitte von Egger und Passarge miteinander zu vergleichen. Zuerst ein Zitat aus Anch'io sono pittore und dann ein Zitat aus Passarges Morphologischen Studien von 1955, wobei ich die Abweichungen unterstreiche:

157. Das zwischen die Blöcke eindringende Regenwasser sammelt sich unter mir an; es entsteht ein Netzwerk von Rieselwasserrinnen: sie sammeln sich unter / dem Blockmantel in kleinen Rinnsalen an, die am unteren Rand des Blockschuttmantels im Joch zu der Unebene der Satteltäler als winzige Erosionsrinnen heraustritt / en, die sich zu geäderten Wasserrissen veruneinigen.

(Asp)

Das zwischen die Blöcke eindringende Regenwasser sammelt sich unter ihnen an; es entsteht ein Netzwerk von Rieselwasserrinnen. Diese sammeln sich bereits unter dem Blockmantel in kleinen Rinnsalen an, die am unteren Rande des Blockschuttmantels im Übergang $\underline{\text { zu der Ebene des Schellaltales als kleine Erosionsrinnen heraustreten, die sich zu größeren }}$ Wasserrissen vereinigen.

(Passarge 1955, 28)

Auch weitere Stichproben zeigen, dass Egger die Übergänge zwischen seinem Text und den aus Passarge übernommenen Passagen nie durch Anführungsund Schlusszeichen kennzeichnet, sie vielmehr zusätzlich verwischt, indem er die Anschlüsse verändert, etwas hinzufügt oder allzu technische Bezeichnungen weglässt. Er bringt zudem Sätze zusammen, die bei Passarge an verschiedenen Stellen stehen. Besonders tiefgreifend ist, dass in obenstehender Passage bei Egger aus »ihnen«, d. h. den Blöcken, ein »mir« wird und damit wiederum eine

21 Louis [1959]. Vgl. zu Siegfried Passarge auch die jüngere Antisemitismus- und Kolonialismusforschung, bspw.: Davis 2012; Berg 2014, 54, 192, 241 sowie Berg 2007, 147-149. 
Übertragung auf das >Ich immer auch das >Ich`meiner Lektüre ist. Passarges Abschnitt ist dem Egger'schen Text zudem ähnlich gemacht, indem bspw. im obigen Abschnitt ein Verb wie ıvereinigen` durch ein für Egger typisches ıver-un-einigen`verändert wird.

Das gleiche kompilatorische Verfahren wendet Egger auch bei der 1933 erschienenen Publikation Wissenschaftliche Ergebnisse einer Reise im Gebiet des Orinoco, Caura und Cuchivero im Jahre 1901-02 an. Unter den übernommenen und teilweise angepassten Abschnitten befinden sich auch längere identische Passagen, wie etwa die Folgende (Passarge 1933a, 168):

165. Wir stehen in dem Wald, der licht ist, so daß man ohne Schwierigkeit in ihm gehen kann. Einzelne, / mannsdicke Bäume verschwinden gegenüber dem Gewirr armdicker Stämme, niedriger Palmenbüsche, schlanker, großblättriger Stauden. Hier dringt in ganzer Fülle / das Licht ein; man ist geblendet. Dort versucht das Auge vergeblich das Dunkel zu durchdringen, hier kommt man raschen Schrittes vorwärts, anderswo hemmt Unt / erholz oder ein umgestürzter Stamm den Fuß. Weich, schwärzlich, humos ist der Boden, auf dem Haufen von Blättern und Zweigen vermodern.

(Asp)

Unter den Büchern, die täglich mit Amazon in die Villa Massimo geliefert wurden, befanden sich also auch diejenigen von Siegfried Passarge - und sie liegen nun, wenn auch nur leihweise aus der Bibliothek, auch auf meinem Schreibtisch. Es stellt sich mit ihnen eine Ge-Wohnheit ein, die ich aber nicht wollte. Ich blättere in ihnen und entdecke bei der Publikation zur Orinoco-Reise schnell, dass darin nicht >nur die geologischen Passagen und Reiseeindrücke enthalten sind, die Egger verwendet, sondern auch ein Landeskundlicher Teil. Ich greife zwei Passagen heraus:

Außer der Landschaft hängt die Kultur in einem Lande von dem Volke und seiner rassenmäßigen Zusammensetzung, ferner von der Kulturstufe und von dem durch die geschichtlichen Ereignisse bedingten Erbgut ab.

(Passarge 1933a, 265)

Ist die geringe kulturelle Leistungsfähigkeit der Mischlinge, die sich auf politisch-wirtschaftlichem Gebiet in der Form der dauernden Revolutionen und des Ausbeutungssystems der jeweiligen Regierungspartei deutlich genug dokumentiert, eine Folge des Unterganges der weißen Rasse, oder liegen andere Gründe vor?

(Passarge 1933a, 268)

Die Handlungen, zu denen mich also Eggers Arbeitsjournal nun veranlasst, bestehen nicht mehr darin, den vom Klang ausgelösten Assoziationen nachzugehen, sondern darin, philologische Vergleiche anzustellen und zu versuchen, Zusatzinformationen und Kontexte an den Text heranzutragen.

Die Veränderung meiner Leserin-Position lässt sich an einem Wort festmachen: Ich bin auf der ersten Seite von Eggers Heft auf das Wort >Wollsackschwinden gestoßen, das zunächst wiederum eine assoziative Reihe in meiner Vorstellung ausgelöst hat, dessen Anführungs- und Schlusszeichen mich jedoch, 
wird damit doch gewöhnlich ein Zitat markiert, zur philologisch-diskursanalytischen Recherche veranlassen - wobei sich herausstellt, dass es Teil eines Abschnitts von Passarge ist und er dieses Wort hervorhebt, weil er damit einen uneigentlichen Ausdruck verwendet. Andere Signale gibt mir der Text nicht - keinen Hinweis darauf, was in ihm auf welche Weise enthalten ist und wieso.

Hier gelange ich auf ganz andere Weise als zuvor an die Grenzen meines Verständnisses. Denn während Eggers Texte die Frage, was Verstehen ist und wie es zustande kommt, ständig aufwerfen, so komme ich hier gerade mit den oben beschriebenen Methoden, mich dem Text anzunähern, nicht weiter: Warum ist es unverzichtbar, gerade Texte von Passarge zu integrieren? Diese Frage stelle ich Oswald Egger und im schriftlichen wie mündlichen Austausch erhalte ich die im Prolog angeführten Informationen und noch einige mehr: Dass es sich bei dem mir vorliegenden Text um ein Arbeitsjournal handelt, dass der Text absichtlich unlesbar gemacht wurde, um die Forderung seitens der Stipendiengeber nach Dokumentation zu untergraben - kurzum, dass es sich also um eine Textmasse handelt, in die eigener Text und Lektüren gleichermaßen eingegangen sind, aber noch nicht zu einem abgeschlossenen Text, wie er bei Suhrkamp publiziert würde, bearbeitet wurde. Ich erfahre, dass ihn bei Passarge mehr der Orinoco interessiert habe als der auch vorhandene biographische Zusammenhang, auf den er mich aber dennoch hinweist: Passarge sei einer jener »deutschen Nazi-Touristen« gewesen, die in Eggers Südtiroler Heimatorten >gewirkt « und zusammen mit »örtlichen Verbündeten« seine Familie »deutlichst schikaniert« hätten. Von diesen persönlichen Geschehnissen kann ich nichts weiter wissen, aber wohl von dem politischen Kontext, in dem sie stehen. Eine weitere Recherche führt mich zu Passarges ebenfalls im Jahr 1933 publizierter Einführung in die Landschaftskunde, in welcher tatsächlich Tscherms und Lana Gegenstand ausführlicher Betrachtungen sind. An vielen Stellen dieser Schrift wird deutlich, welch fatale Konsequenzen die Grundannahme Passarges haben, einen `Zusammenhang` zwischen Natur, Kultur, Tier und Mensch herstellen zu wollen. ${ }^{22}$ Das Stichwort der `Natur` und >Natürlichkeit` dient einmal mehr der Auf- und Abwertung bestimmter Menschengruppen. So gebe es eine »Überbevölkerungsgrenze« der Landschaft, die, wie etwa in Nordamerika, in Einklang mit der »Kulturstufe« der Amerikaner stehe und durch Einwanderung aus dem Gleichgewicht geraten könne: »Man besiedle diese Länder mit Chinesen, und die Bevölkerungszahl würde gewaltig emporschnellen.« (Passarge 1933b, 86) Passarge bedient auch in dieser Schrift wahllos antisemitische

22 Passarge 1933b, 82: "Die Landeskunde behandelt also in erster Linie die Abhängigkeit der kulturellen Verhältnisse (Wirtschaft, Siedlungen, Verkehr, materieller und geistiger Kulturbesitz, soziale und politische Verhältnisse) und die der Bevölkerung (Völker, Charakterbildung, Begabung, Volksdichte) von dem künstlich begrenzten Landraum (meist Staatsraum).« 
Topoi (Passarge 1933b, 88), politisiert gegen Russland, die Tendenzen zur Auflösung von Familie und Ehe etc. Am Ende folgt die Diagnose von »dekadentsenile[n] Zustände[n] « der Gegenwart und das Fazit, dass »Rettung [...] höchstens eine aus den gesunden, noch nicht degenerierten Schichten des Volks aufquellende Bewegung bringen [könne], die ein das ganze Volk einendes, den Klassenhaß bekämpfendes, kollektivistisches Nationaldenken den Kräften des demoralisierenden Materialismus, Egoismus und Parteidenkens siegreich gegenüberstellt.« (Passarge 1933b, 91) Dass mit dieser Haltung unter dem Deckmantel der Wissenschaft auch koloniale Absichten verbunden sind, wird ebenfalls explizit formuliert -und zwar auf das Südtirol bezogen, dessen Bedeutung als Rohstoffquelle er hervorhebt: Weil »jeder Staat nach Autarkie streben« müsse, müsse auch »das deutsche Mitteleuropa nach Südtirol und dem Elsaß als nach Ergänzungslandschaften verlangen. Beide Gebiete gehen in dem jetzigen `Landverband (=Staatsverband) wirtschaftlich zugrunde, als Teile Deutschlands bzw. Österreichs blühen sie auf.« (Passarge 1933b, 85)

Die Auseinandersetzung mit Passarge kann für Egger also eine Beschäftigung mit seiner Familiengeschichte und der Geschichte der Gegend, in der er aufgewachsen ist, sein - er thematisiert diesen Komplex bspw. auch in dem Essay Deutscher sein (Egger 2013). Sein Vater kommt darin am Rande vor als jemand, der sich als >Paschgänger`, d. h. als Schmuggler, durchs Gebirge bewegte, jedoch laut dem Briefgespräch >fast Illiterat` (Egger im Interview mit Braun [2000]) keine Stimme hat. In dem Austausch zwischen Egger und mir geht es u. a. auch darum: Wer hat eine Stimme? Wieso hat Passarge eine Stimme, Eggers Eltern aber nicht? Wer etwas sagt, was gesagt und was nicht gesagt ist - das sind zentrale Themen von Eggers Poetik. Was nicht gesagt ist, ist der Titel sowohl einer ebenfalls im Jahresbericht einsehbaren Rede an der Villa Massimo - ich habe daraus bereits zitiert - als auch seiner Berliner Rede zur Poesie (Egger 2016).

Seit ich all dies weiß, kann ich meine Erschütterung darüber, unvermittelt auf solche ideologischen Textbausteine gestoßen $\mathrm{zu}$ sein, etwas besser einordnen und differenzieren. An diesem Arbeitsjournal werden die Grenzen einer rein immanenten Auseinandersetzung mit literarischen Texten ebenso deutlich wie die Bedeutung der Kontexte. Ich konnte den Autor um einige Hinweise bitten, die mir weiterhelfen, aber freilich nur begrenzt, denn am Anfang und am Ende habe ich doch einfach den Text, so wie er vor mir liegt. Als Leserin, die nicht gerade einen Aufsatz für den vorliegenden Band schreiben sollte, hätte ich ihn, spätestens mit der Entdeckung der Passarge-Abschnitte, vielleicht schon früher, einfach weggelegt. »Ich könnte sie lesen, aber ich will nicht - der Satz von Ann Cotten, der am Anfang meiner Überlegungen stand, hat seine Bedeutung grundsätzlich verändert. Ich hätte einfach keine Lust mehr gehabt, weiterzumachen - denn, so fragte ich auch Egger, wie kann ich wissen, was nicht gesagt 
ist? Von der - offenbar intentional hergestellten - schweren Lesbarkeit, die stets droht, meine Schritte durch den Text zu ersticken, bis hin zu der unerwarteten Konfrontation mit Passarge: Der Text baut zusätzlich zu den Schwellen, die Eggers Texte stets erfahrbar machen, zusätzliche Hindernisse auf, für die ich kaum Verständnis aufbringen kann. Aber ich nehme doch noch einen letzten Anlauf, verbunden mit der Frage:

\section{Warum lesen?}

Dass es sich bei Anch'io sono pittore um ein Arbeitsjournal handelt, das nicht für eine breite Rezeption gedacht war, gibt diesem Text einen besonderen Status. Und doch ist es ein gestaltetes, ein gedrucktes Heft, das randvoll mit Buchstaben auf meinem Schreibtisch liegt.

Es mag sich um einen zufällig möglich gewordenen Blick in eine für einen spezifischen Kontext präsentierte >Werkstatt handeln. Aber auch ein Arbeitsjournal kann Auskunft über die poetischen Verfahren eines Schriftstellers geben, vielleicht sogar bestimmte Aspekte deutlicher hervortreten lassen, und so komme ich nochmals darauf zurück, weshalb ich von meiner Entdeckung der Passarge-Zitate so schockiert war und bei aller Differenzierung noch immer bin.

Zunächst ist die Art und Weise, dieses Arbeitsjournal zu gestalten, aufschlussreich: Im Vergleich mit den anderen Dokumentationen der Stipendiat*innen, die Fotos von Werken in den Ateliers, von Hand korrigierte Textblätter, Erklärungen zum Konzept etc. enthalten und damit den >Werkstattcharakter «, die Quellen, Ideen und Kontexte, explizit thematisieren, geschieht in Eggers Textmasse eine Abdichtung gegen genau dieses Ausstellen eines künstlerischen Prozesses. Er sagt nicht, welche Bücher er bei Amazon bestellt hat, er gibt keinen Hinweis auf das, was ihn in dem Stipendienjahr künstlerisch beschäftigt hat, sondern gibt >es` schwer lesbar zu lesen. Diese Verweigerung mag, wie er es mir gegenüber formuliert hat, auf die sLeistungsschau <ezogen sein, die mit solchen Stipendien verbunden ist, aber es kommt darin auch eine Haltung zum Ausdruck, die keinen Wert darauf legt, von der kommunikativen Funktion von Sprache und den Konventionen im Umgang Gebrauch zu machen, um bspw. die Passagen eines Autors mit rassistischen Ideologien $\mathrm{zu}$ problematisieren oder zumindest $\mathrm{zu}$ markieren. Den Konsequenzen dieser Sprache, die als totale Rede alles in sich integriert, ohne zu differenzieren, hatte ich mich zu stellen, als ich nicht weiterkam mit dem Heft, so wie es sich mir zufällig präsentierte, und - was nicht ohne Ironie ist - dann das Gefühlt hatte, den Autor doch um einige Hinweise zu bitten und damit einzufordern, was der Text selbst mir vorenthielt. 
Die sich mir aufdrängenden materiellen und hermeneutischen Schwellen standen am Anfang meiner Auseinandersetzung mit dem Heft Anch'io sono pittore und sie haben grundsätzlich etwas mit Eggers Poetik zu tun, die sich von der >Verschwendung an die Verständigung، abwendet. Ich habe auf diese Schwellen mit dem Versuch reagiert, mich ganz in das Treiben des Textes hineinzubegeben, mit meinen körperlichen Handlungen, meinen Assoziationen und auch meinen Emotionen. Ich bin zum lesenden Kind geworden, das sich ganz in die kreisenden Bewegungen des Textes hineinbegeben hat - ihn berührt und sich ihm ähnlich gemacht hat. Ich habe versucht, das Wörterbuch als ebenso hermeneutisch-philologisches wie auch sinnliches Instrument der Lektüre einzusetzen.

Warum also Egger lesen? Die beglückendsten Momente bei der Beschäftigung mit dem Text bestanden darin, mich in das Klang-Bad hineinzubegeben, die Bewegung der Sprache nachzuvollziehen, mich den eigenen Assoziationen anheimzugeben und im Dialog mit den Wörterbüchern - die die Mehrdimensionalität von Wörtern so greifbar werden lassen - zu erfahren, dass ein gleichzeitiges Verstehen und Nicht-Verstehen möglich und als solches beschreibbar ist. Spaß - und ja, `Glückı und `Spaß sind für mich relevante Lesekriterien - macht es immer dann, Egger zu lesen, wenn ich mit meinem Finger in relativ hohem Tempo über eine Paßage hinwegfahre und diese Erfahrung zwischen Verstehen und Nicht-Verstehen mache, die ich zunächst beobachten und sodann auch genauer analysieren kann. Ich bin bei der Lektüre auf mich selbst zurückgeworfen, weil ich mich selbst, meine Handlungen, meine Gefühle und Vorstellungen einbringen muss. ${ }^{23}$

Felix Philipp Ingold hat eine gewisse Ermüdung festgestellt in seiner Beschäftigung mit den Texten Eggers, weil sie, wie er meint, nach dem immergleichen Prinzip funktionieren (Ingold 2016). Und tatsächlich könnte die eben beschriebene Erfahrung auch mit allen anderen Texten Eggers gemacht werden. Eggers Texte sind in dem Sinne Meta-Literatur, als sie Fragen in der Rezeption aufwerfen, die andere Texte auch, aber vielleicht in weniger herausgehobener Weise stellen, und entsprechend haben Eggers Texte auch etwas Manieriertes. So sehr ich dieses Tempo mag, das sich aus dem kreiselnden Sog entwickelt - ich fühle mich bisweilen, auch weil ich ja in der Mitte dieses geschlossenen TextKreises sitze und mich darin unablässig um meine eigene Achse oder den Text um mich herum drehe, wie auf einem Karussell: Ein paar Runden machen Spaß, aber irgendwann wird mir schlecht und ich möchte wieder raus. Eine

23 Eleonore de Felip $(2013,52)$ spricht von >Allegorien des Lesens`, versteht darunter jedoch etwas anderes, als hier gemeint ist. 
Besessenheit oder eben: Wut - wie sie der Text als poetologisches Verfahren anführt - fällt dann von mir ab. ${ }^{24}$

Wenn nun in Anch'io sono pittore Abschnitte aus Publikationen von Passarge auftauchen, ist die Problematik darüber hinaus noch einmal anders gelagert. Ich habe oben bereits darauf verwiesen, dass die Anthropomorphisierungen der Natur ebenso wie das vereinzelt erscheinende >Ich`zentral sind für die Aneignung des Textes und die Übertragung der affektiven Zustände auf mich selbst, also für die mimetischen Handlungen, zu denen mich der Text veranlasst. In dem Moment, wo geologische Passagen eines nationalsozialistischen Landschaftsgeographen das sind, worauf ich - zunächst unwissend - meine affektiven Energien richte, geht es nicht mehr um >Sprachlandschaften` und um meine Assoziationen und Gefühle, sondern es geht um ganz konkrete Landschaften und die Frage ihrer ideologisch geprägten - Beschreibung. So vielfältig, ja im Sinne von >Achilles` allumfassend, ${ }^{25}$ jenes $>$ Ich เ bei Egger überhaupt und auch in Anch'io sono pittore sein mag: Es ist hier auch das >Ich` von Passarge.

Blicke ich nochmals auf die oben angestellten Vergleiche zurück, dann ist es einerseits so, dass sich das Egger'sche Ich in den Text von Passarge hineinschleicht, der durch Entkontextualisierung, Zusammenschneiden, Ergänzen und Verändern sunlesbarı gemacht und ein Stück weit seiner Intention sowie seiner Handlungsfähigkeit entmächtigt wird. Gleichzeitig aber macht dieses >Ich`sich dem Text von Passarge ähnlich und es erlaubt ihm, in den eigenen Text einzuwandern und auch in die Übertragungsprozesse auf mich hineinzuwirken. Besonders offensichtlich ist dies an denjenigen Stellen, wo wie in der Orinoco-Reisebeschreibung ein explizit persönliches >Wir s spricht oder wo Egger einen Gegenstand - die Felsblöcke - durch ein >Ich، ersetzt. Dieser Vorgang gleicht meinem mimetischen Leseverfahren und verunmöglicht es genau in diesem Moment. Denn hier werden die Grenzen zwischen den Stimmen tatsächlich verwischt - mit der Folge, dass auch ich zu eben jenem >Ich « werde, das ebenso Egger wie auch Passarge ist. Es ist mehr als eine seltsame >Gleichzeitigkeit des Ungleichzeitigen ${ }^{26}{ }^{26}$ dass Passarges Einführung in die Landschaftskunde und seine Orinoco-Beschreibung im gleichen Jahr (1933) erschienen sind wie Walter Benjamins Text über das Schmökern. Wenn das, von dem ich mich so sehr durchschneien und einschneien lasse, ohne dass es

24 Vgl. hierzu auch Andrea Albrecht (2017, 229): „Das topologische Bild der Sphärenumstülpung aufgreifend, beschreibt Egger seine über Hamann geführte Einsicht in die rein sprachliche, jedenfalls nicht mehr religiös getragene Existenz des Menschen als radikalen, weil ihn tendenziell isolierenden, den Text >dicht machenden` Sturz nach Innen.»

25 >Achilles` kann als Kombination von sich

26 Vgl. zu dieser u. a. von Ernst Bloch geprägten Gedankenfigur: Schneider und Brüggemann 2011. 
mir signalisiert wird, ein Text ist, der in Teilen selbst von einem Text durchdrungen ist, der völkische und rassistische Ideologien verbreitet, dann entzieht sich mir - ein anderes >Ich « wäre diesem Subtext vielleicht anders gegenübergestanden - das Vertrauen in den gesamten Text: Gerade die mimetische Angleichung, die die poetischen Verfahren von Anch'io sono pittore auch aufgrund der Hermetik eingefordert haben, werden für mich zum Problem - denn es macht einen Unterschied, welchen Buchstaben ich folge, um sodann meinen affektiv-bilderreichen Assoziationen nachzuhängen und an welchen Text ich meine Einbildungskraft und Phantasie verschwende. >Transvaalı liegt eben nicht nur, wie bei Benjamin, im Inneren des lesenden Kindes, sondern weiterhin auch im Außen, als eine der Provinzen Südafrikas, auf die am Ende des neunzehnten Jahrhunderts die Deutschen Kolonialbestrebungen gerichtet waren und die in diesem Zusammenhang auch von Siegfried Passarge bereist wurde (Louis [1959]).

Dabei wird im Fall von Passarge vielleicht auch nur besonders deutlich, was überhaupt als poetisches Verfahren von Egger in Hinblick auf die ihm impliziten politischen Strategien befragt werden kann: Was passiert, wenn so grundsätzlich nicht zwischen eigenem und fremdem Text unterschieden, wenn der fremde Text in den eigenen integriert, ihm ähnlich gemacht wird? Und was passiert, wenn meine Stimme und die des Textes, das Innen und Außen, nicht mehr getrennt werden können? Was bedeutet es, wenn das Ich alles und nichts zugleich ist? Ganz zu Beginn der Berliner Rede zur Poesie heißt es:

Ich starre Raster in den Tag, die Grenzen der Grenzen verwischen und ereignen einander als in sich kreisende Welten in der Welt, rede ich mir ein, die bis zum Dort hinaus, ja, aber auch über das Dort hinaus?

(Egger 2016, 7)

Ich kann diese Stelle nicht vertieft analysieren, doch scheinen hier sogar die Grenzen der Grenzen zu verwischen, es gibt sin sich kreisende Welten`, aber ob dieses Kreisen auch zu einem Dort und über dieses hinaus geht, wird als Frage gestellt. Man hat versucht, solche Stellen politisch zu deuten - in einem Interview wird gefragt, ob sie auf die `Flüchtlingsfrage ` bezogen seien. Egger gibt darauf zur Antwort, »noch nie etwas anderes getan [zu haben] als politische Lyrik«, jedoch wiederum konkret auf die Sprache bezogen, die nie eine bestimmte Position innehat, mit dem Hinweis, dass das Wort »Grenze« für den »Parteigänger«, den »Paschgänger« und den »Mathematiker« etwas anderes bedeuten (Egger im Interview mit Schwazer 2017).

Eine >Politik der Literatur kann darin bestehen, die Worte ihren üblichen Registern zu entziehen und im Text eine andere >Aufteilung des Sinnlichen ` wie Jacques Rancière (2006) es formuliert - herzustellen. So erstaunt es nicht, dass Egger auf die Interview-Frage nach dem >Ich` in seinen Texten auch genau 
das beschreibt, worauf ich oben im Zusammenhang mit Benjamin verwiesen habe:

Ich war schon Knabe, Mädchen, Pflanze, Vogel und flutentauchender, stummer Fisch, dies kann man bereits aus Empedokles übersetzen, dem ersten Dichter der verlorenen Läuterungen. Und mein Nonstal sollte auch so ein Läuterungsberg begrenzen, der Idee nach, ein Ungelände quasi, bei dem Grat um Grat gleichsam unter die Grammel kommt. Insofern gibt es keine Grenzen, kein Ich, und kein inzwischen gewichtetes Gedicht, davon. $^{27}$

(Egger im Interview mit Schwazer 2017)

Auch hier ist das >Ich nicht, wie es der Interview-Kontext vermuten lässt, Eggers Stimme, sondern eine Übersetzung eines Empedokles-Fragments. In diesem Allesund zugleich Nichts-Sein verweist Egger - passend zu meinen Assoziationen an die Genesis und antike mythologische Erzählungen - auf dessen Lehre des Ähnlichen und den Läuterungsberg, wie er etwa als Fegefeuer in Dantes Divina Commedia dargestellt ist. Er benennt damit wiederum zwei mystisch-religiöse Kontexte, deren Vorstellungen von Totalität er auf seine Rede bezieht, bildet sich doch in Anch'io sono pittore ein Strom ohne Unterbrechung, in dem es keine räumlichen und zeitlichen Differenzen gibt. Zusammen mit den zirkulär-tautologischen Strukturen formiert dieser kontinuierliche Schriftstrom, der alles in sich einspeist, letztlich einen in sich geschlossenen Kreis. Stellt sich in dieser Auflösung der Grenzen eine Gleichheit ein? Oder ist mit dieser Integration von allem und jeder ${ }^{\star} \mathrm{m}-$ die sich auch auf Passarge erstreckt - auch eine Gleichgültigkeit verbunden gegenüber der Frage, welche Darstellungsform für die Auseinandersetzung mit einem antisemitisch-rassistischen Text adäquat ist?

Einer `Ethik der Lektüre` zu folgen, wie es dieser Band als Aufgabe den Texten gegenüber einfordert, heißt einerseits, nicht einfach Schlüsselstellen zu wählen, „um dann durch kulturwissenschaftliche Narrative den Raum zwischen ihnen flugs $\mathrm{zu}$ verfugen ${ }^{28}{ }^{28}$ Verbunden ist damit auch die Notwendigkeit, in den ununterbrochenen Textfluss einzuhaken und dort die schnellen Verfugungen und unkenntlich gemachten Fügungen genauer anzuschauen. Eine solche Philologie die mit Blick auf Passarge auch fragen muss: warum? - führt mir vor Augen, dass das Arbeitsjournal Anch'io sono pittore blind ist gegenüber diesen Verfugungen und ihren Konsequenzen. In den verwischten Grenzen zwischen dem eigenen

27 Identischer Wortlaut in Eggers Herde der Rede II. »Ich war schon Knabe, Mädchen, Pflanze, Vogel und flutentauchender, stummer Fisch « ist ein Zitat aus einem Fragment von Empedokles, vgl. Kranz 1992, 359, Fragment 117. Fast identisch (»Ich zum Beispiel war schon Knabe, Mädchen, Pflanze, Vogel und Flut-enttauchender Fisch.«) bereits in Herde der Rede II (Egger: Herde der Rede II).

28 So eine Formulierung von Martin Endres und Ralf Simon in einem Programmpaper für diesen Band (s. hier auch die Einleitung). 
und dem fremden Text wird vor allem deutlich, dass das `Dort jenseits der in sich kreisenden Welten nicht in den Blick kommt. Es findet keine Verwandlung des >Ich`statt und damit auch keine Relativierung des Subjekts, sondern im Gegenteil wird dieses >Ich 'gerade überhöht - weil es keine bestimmte Stimme bezeichnet, wird alles darauf bezogen, alles integriert. Darin gründet der Schwindel, den die Lektüre bei mir auslöst, die die Projektion meiner Affekte auf die angedeuteten Landschaften erfordert.

Es muss mit Blick auf diese in sich kreisenden Welten also auch gefragt werden, worum es in Eggers Texten eigentlich geht. Es gibt in ihnen keine konkreten Gegenstände, keine Handlungen im Sinne von Interaktionen, keine Figuren und keine Geschichte. Aber so selbstreferenziell sie auch sind, so ist der Bezug zur Natur bzw. dem Naturschönen in ihnen nicht ganz gekappt. Anders als bei anderen experimentellen Richtungen - bspw. im (Neo-)Dadaismus ${ }^{29}$ oder der Wiener Gruppe -, die jegliche Form von Mimesis verabschieden, hat ein Text wie Val di Non allen weiteren Konnotationen zum Trotz tatsächlich ein reales Tal im Südtirol bereits dem Titel nach zum Gegenstand. Auch wenn es mir vollkommen verfehlt erscheint, einen solchen Text, wie Björn Hayer (2018) dies getan hat, aufgrund der Anthropomorphisierungen der Natur in einen Zusammenhang mit einem New Materialism zu stellen, der die nichtmenschlichen Akteure stärker ins Zentrum rückt, so sind die Evokationen von Landschaften, so wie ich sie zu Beginn dieses Beitrags analysiert habe, doch mehr als sinwendige Landschaften Sprachräume (De Felip 2013). In allen Texten Eggers geht es um Vorstellungen, die aber doch auf Landschaften bezogen sind - dies wird in Anch'io sono pittore besonders evident, wo mit Passarges Beschreibungen ganz explizit konkrete historische Landschaften präsent sind: Wie kann eine Sprache, die sich so radikal der kommunikativen Funktion entzieht und sich dem Paradigma der >Beschreibung verweigert, gleichzeitig doch >Natur` zum Gegenstand haben? Wie stellt sich das Verhältnis von Natur und Text hier dar? Siegfried Passarge geht von einer >Lesbarkeit` der Landschaft aus, um Bezüge zwischen der Geographie und der >Kulturstufe der Menschen herstellen zu können. Trotz der >Wissenschaftlichkeit bewegen sich seine Texte, bspw. wo wie in der oben zitierten Stelle das subjektive Erleben des `Malerischen im Register des Naturschönen. ${ }^{30}$ Egger macht freilich etwas ganz anderes, aber auch der von ihm angeführte Bezug seiner Texte zur Empedokles'schen Ähnlichkeitslehre sowie das `malerische` Vokabular der Linien, Schnörkel und Kleckse in

29 Stahl (2017) ordnet Egger dieser Richtung zu, was ich jedoch zumindest teilweise kritisch sehe.

30 Vgl. bspw. die Beschreibung der Hell-Dunkel-Kontraste in der oben zitierten Stelle. 
Anch'io sono pittore erscheinen mir als Reminiszenzen der romantischen Vorstellung, die Hieroglyphen der Natur entschlüsseln zu können. Nur wird gerade am Umgang mit den ebenso konkret historisch wie geographisch verorteten Landschaften, die in Passarges Worten auch in Eggers Text präsent sind, deutlich, dass es ihm gerade nicht um die >Natur geht. Anstatt zwischen der Natur und ihren verschiedenen Beschreibungen zu unterscheiden, werden die Grenzen verwischt, mit dem Resultat, dass gerade keine Öffnung zur Natur hin geschieht, sondern - ganz der Kategorie des Naturschönen entsprechend - eine Abdichtung gegen sie stattfindet, die es dem Text ermöglicht, selbst zu einer Meta-Landschaft zu werden.

Eggers Texte sind keine >Prosa`, die sich dem Bann der Natur und dem Mythos entzieht, sondern sie gehören vielmehr eben jenem in die Imagination transportierten, dadurch vielleicht abgegoltenen, aber nicht aufgelösten Mythos $\mathrm{zu}$, der mit dem `Naturschönen` verknüpft ist - wie es insbesondere Theodor W. Adorno herausgearbeitet hat, dessen Essay über Engagement und Lyrik ich in den letzten Wochen unterrichtet habe und der mir deswegen gerade besonders präsent ist (Adorno 2003a, 104f.). In den tautologischen, zirkulären und zyklischen Strukturen, wie ich sie auf verschiedenen Ebenen bei Anch'io sono pittore festgestellt habe, entsteht - zusammen mit den Assoziationen an die Genesis und andere mythische Erzählungen - eine von historischer Zeit und räumlicher Verortung gänzlich losgelöste (Text-)Landschaft, die ihre Herkunft noch dort unkenntlich macht, wo sie sich mit Passarge in einem spezifischen ideologischen Kontext bewegt. Der Text wird selbst zu einem chiffrierten >Buch der Natur mit unzähligen Sternen, aus denen ich mir - so Eggers eigenes poetologisches Bild - die Sternbilder zusammenlesen soll, freilich mit dem Unterschied, dass es hier, anders als bei Benjamin, nicht mehr um eine alte mimetische Praxis geht, sondern allein die eigenen Projektionen zur Disposition stehen.

Lyrik erhofft sich, nach Adorno, von »rückhaltloser Individuation [...] das Allgemeine«, aber ihr »Risiko« besteht darin, »daß ihr Individuationsprinzip nie die Erzeugung von Verpflichtendem, Authentischem garantiert. Sie hat keine Macht darüber, ob sie nicht in der Zufälligkeit der bloßen abgespaltenen Existenz verharrt.« (Adorno 2003b, 50) Genau diese Frage stellt sich hier - ganz unabhängig von der Frage, ob man es mit Lyrik oder Prosa zu tun hat -, wo mit Passarge noch das Fernstehendste in den subjektiven Strudel hineingezogen wird und die Grenzen aufgehoben werden. Versteht man Anch'io sono pittore im Sinne des Arbeitsjournals tatsächlich als Auszug aus einem täglichen Schreibpensum - mit Bezug auf Peter Handkes Mein Jahr in der Niemandsbucht hat Egger einmal angegeben, jeden Tag eine Seite zu schreiben (Egger im Interview mit Klein 2010) -, das dann weiter bearbeitet und destilliert wird und irgendwann zu einem Buch führt, dann kann man Eggers poetisches Verfahren insgesamt als einen ununterbrochenen Schreibfluss verstehen, als ein ständiges Textweltenbauen, dessen Re- 
sultate ab und zu in ein Buch gebracht werden. Sie sind sich selbst genügsam man kann mal shineinschnäuggen`, man muss aber nicht.

Denn es gibt einen Punkt in dieser Poetik, an dem das Zirkuläre, das Selbstreferenzielle, die Negation - als Verweigerung, auf etwas hinzudeuten - selbst ein affirmatives Element erhalten. Dies hat Adorno in seinem Versuch, das autonome Kunstwerk sowohl gegen ein gut gemeint Engagiertes als auch gegen ein rein Ästhetizistisches zu verteidigen, mit Bezug auf das letztere formuliert: »[W]as die letzte Kommunikation durchschneidet, wird zur Beute der Kommunikationstheorie. Kein festes Kriterium zieht die Grenze zwischen der bestimmten Negation des Sinnes und der schlechten Positivität des Sinnlosen als eines beflissenen Weitermachens um seiner selbst willen." (Adorno 2003b, 426) Es gibt keine sfeste Grenze`, aber diese Grenze in den Blick zu nehmen, scheint mir doch wichtig. Ich möchte mich dem, was im »Schock des Unverständlichen passiert«, aussetzen und die Rebellion gegen die "positive Unterstellung von Sinn« anerkennen (Adorno 2003b, 412). Aber ich möchte mich nicht in eine totale Rede hineinbegeben, die den Bezug zur Welt evoziert und dann gleich wieder negiert, aber mich gerade dort im Stich lässt, wo sie selbst den Bezug zu einer ganz spezifischen historischen Realität und einer sprachlichen Politik herstellt, ja von dieser Realität materiell durchdrungen ist.

Nachdem sich der Text dieser Verantwortung entzogen hat, erscheint mir mein Versuch, mich seinem >Treiben` auszusetzen, der mir doch gerade als die einzige Möglichkeit erschienen war, mich diesem Text überhaupt zuzuwenden, hinfällig, ja mehr noch: durchaus problematisch.

Also nochmal: Warum Egger lesen? Es ist eine körperliche und affektive Erfahrung von Sprache, die mich ganz auf mein Lesen als einen eigenen Raum und eine eigene Zeit zurückführt. Dabei stellt sich das vom Text eingeforderte Lesen als mimetische Angleichung durch die Konfrontation mit Passarge zwar als eine Möglichkeit, aber als eine unmögliche Möglichkeit heraus. ${ }^{31}$ Es geht also darum, die Grenzen wieder einzuziehen, die Anch'io sono pittore negiert: Es mag dann sein, dass Eggers Text die Grenzen dieser meiner Leserin-Position sichtbar macht. Aber die Grenzen meiner Lektüre sind auch die Grenzen des Textes selbst.

31 Vgl. hierzu Derrida 2003. 


\section{Literaturverzeichnis}

Adorno, Theodor W.: Ästhetische Theorie. Frankfurt a. M. 2003 a.

Adorno, Theodor W.: Noten zur Literatur. Frankfurt a. M. 2003b.

Adorno, Theodor W.: Über Walter Benjamin. Hrsg. und mit Anm. versehen v. Rolf Tiedemann. Frankfurt a. M. 1970.

Albrecht, Andrea: "Auszug aus selbstverschuldeter Verständlichkeit«. Hermeneutisches und Hermetisches zur Poesie Oswald Eggers. In: Scientia Poetica. Jahrbuch für Geschichte der Literatur und Wissenschaften 21.1 (2017), 210-235.

Anzieu, Didier: Das Haut-Ich. Übers. v. Meinhard Korte und Marie-Hélène Lebourdais-Weiss. Frankfurt a. M. 1991.

Benjamin, Walter: Gesammelte Schriften. Unter Mitwirkung von Theodor W. Adorno und Gershom Scholem hrsg. v. Rolf Tiedemann und Hermann Schweppenhäuser. 14 Bde. Frankfurt a. M. 1991.

Berg, Nicolas: Racism and Anti-Semitism in the German Political Economy: The Example of Carl Schmitt's 1936 Berlin Conference »)ewry in Jurisprudence«. In: Ideas of »Race« in the History of the Humanities. Hrsg. v. Amos Morris-Reich und Dirk Rupnow. London 2007, 139-158.

Berg, Nicolas: Luftmenschen. Zur Geschichte einer Metapher. Göttingen 2014.

Blüher, Joachim (Hg.): Villa Massimo. Deutsche Akademie Rom. Jahresbericht 2014. Rom 2015. 5. November 2020: https://www.villamassimo.de/content/4-informationen/1-villa-mas simo/3-studienaufenthalt/5-jahresberichte/jahresbericht_2014.pdf.

Brüggemann, Heinz: Walter Benjamin über Spiel, Farbe und Phantasie. Würzburg 2007. Cotten, Ann: Lyophilia. Berlin 2019.

Da Vinci, Leonardo: Das Buch von der Malerei. Hrsg. v. Heinrich Ludwig. Wien 1882.

Davis, Christian S.: Colonialism, Antisemitism, and German Jewish Descent in Imperial Germany. Michigan 2012.

De Felip, Eleonore: Inwendige Landschaften oder Die leeren Räume der Sprache. Peter Waterhouse' "Spaziergang als Himmelskunst « und Oswald Eggers »Im Anger des Achilles«. Möglichkeiten und Grenzen einer wissenschaftlichen Lektüre. In: Studia austriaca 21 (2013), 51-80.

Derrida, Jacques: Eine gewisse unmögliche Möglichkeit, vom Ereignis zu sprechen. Übers. v. Susanne Lüdemann. Berlin 2003.

Deutsches Wörterbuch von Jacob und Wilhelm Grimm. 16 Bde. in 32 Teilbänden. Quellenverzeichnis Leipzig 1971. Online-Datenbank. Leipzig 1854-1961. 5. November 2020: http://dwb.uni-trier.de/de/ [= DWB].

Dudenredaktion: Ungestalt. 5. November 2020: https://www.duden.de/rechtschreibung/ ungestalt.

Egger, Oswald im Interview mit Michael Braun: Über die natürliche Magie der Einbildungskraft. Ein Briefgespräch in zehn Kapiteln mit Oswald Egger und Michael Braun anlässlich der Verleihung des Clemens Brentano Förderpreises für Literatur der Stadt Heidelberg 2000 an Oswald Egger [2000]. 5. November 2020: https://www.heidelberg.de/hd/HD/Rat haus/Interview+mit+Oswald+Egger.html.

Egger, Oswald: »Herde der Rede Moiré«. In: Minima Poetica. Für eine Poetik des zeitgenössischen Gedichts. Hrsg. v. Joachim Sartorius. Frankfurt a. M. 2003, 40-47. 
Egger, Oswald im Interview mit Christina Weiss: »/ch will semantische Wolken erzeugen «. In: Welt Print (27.5.2010). 5. November 2020: https://www.welt.de/welt_print/kultur/arti cle7801662/Ich-will-semantische-Wolken-erzeugen.html.

Egger, Oswald im Interview mit Erich Klein: »Ich weiß immer weniger, was ein Gedicht ist. "In: Falter 28 (2010), 26. 6. November 2020: https://shop.falter.at/detail/9783518421338.

Egger, Oswald: Deutscher sein. Leonberg 2013.

Egger, Oswald: Anch'io sono pittore. In: Villa Massimo. Deutsche Akademie Rom. Jahresbericht 2014. Hrsg. v. Joachim Blüher, Deutsche Akademie Rom Villa Massimo. Rom 2015a [unpag.]. 5. November 2020: https://www.villamassimo.de/content/4-informatio nen/1-villa-massimo/3-studienaufenthalt/5-jahresberichte/jahresbericht_2014.pdf.

Egger, Oswald: Was nicht gesagt ist. In: Villa Massimo. Deutsche Akademie Rom. Jahresbericht 2014. Hrsg. v. Joachim Blüher, Deutsche Akademie Rom Villa Massimo. Rom 2015b, 117-127. 5. November 2020: https://www.villamassimo.de/content/4-informatio nen/1-villa-massimo/3-studienaufenthalt/5-jahresberichte/jahresbericht_2014.pdf.

Egger, Oswald: Berliner Rede zur Poesie. Göttingen 2016.

Egger, Oswald im Interview mit Heinrich Schwazer: Georg Trakl Preis an Oswald Egger. In: Die neue Südtiroler Tageszeitung (18.8.2017). 5. November 2020: https://www.tageszeitung. it/2017/08/18/georg-trakl-preis-an-oswald-egger/.

Egger, Oswald: Herder der Rede II. 5. November 2020: https://www.lyrikline.org/de/gedichte/ herde-der-rede-ii-378.

Endres, Martin: Nach dem Muster. Paradigmatizität der Poesie. In: Paradigmenwechsel. Wandel in den Künsten und Wissenschaften. Hrsg. v. Andrea Sakoparnig, Andreas Wolfsteiner und Jürgen Bohm. Berlin, Boston 2014, 277-284.

Hayer, Björn: Die Stimmen der Natur. Materie, Flora und Fauna - in der Gegenwartslyrik scheint alles im Werden zu sein. In: Neues Deutschland (7.7.2018). 5. November 2020: https://www.neues-deutschland.de/artikel/1093468.die-stimmen-der-natur.html.

Ingold, Felix Philipp: Das Nichts sagen, und noch viel mehr. In: Volltext 3 (2017), 16-19.

Jakobson, Roman: Linguistik und Poetik. In: ders.: Poetik. Ausgewählte Aufsätze 1921-1971. Hrsg. v. Elmar Holenstein und Tarcisius Schelbert, Frankfurt a. M. 1979, 83-121.

Kranz, Walther (Hg.): Die Fragmente der Vorsokratiker. Griechisch und Deutsch v. Hermann Diels. Bd. 1. Hildesheim 1992.

Louis, Herbert: Nachruf auf Siegfried Passarge [1959]. 6. November 2020: https://badw.de/fi leadmin/nachrufe/Passarge\%20Siegfried.pdf.

Mainberger, Sabine: „Der Künstler selbst war abwesend.« Zu Plinius’ Erzählung vom Paragone der Linien. In: Im Agon der Künste. Paragonales Denken, ästhetische Praxis und Diversität der Sinne. Hrsg. v. Hannah Baader, Ulrike Müller Hofstede, Kristine Patz und Nicola Suthor. München 2007, 19-31.

Passarge, Siegfried: Wissenschaftliche Ergebnisse einer Reise im Gebiet des Orinoco, Caura und Cuchivero im Jahre 1901-02. Hamburg 1933a.

Passarge, Siegfried: Einführung in die Landschaftskunde. Leipzig, Berlin 1933b.

Passarge, Siegfried: Morphologische Studien in der Wüste von Assuan. Hamburg 1955.

Rancière, Jacques: Die Aufteilung des Sinnlichen. Die Politik der Kunst und ihre Paradoxien. Hrsg. v. Maria Muhle, aus dem Franz. v. ders., Susanne Leeb und Jürgen Link. Berlin 2006.

Rosa, Hartmut: Vom Wunder narrativer Resonanz. In: Warum lesen. Mindestens 24 Gründe. Hrsg. und mit einer Nachbemerkung v. Katharina Raabe und Frank Wegner. Berlin 2020, 196-217. 
Rosenberg, Raphael: Der Fleck zwischen Komposition und Zufall. Informelle Ansätze in der frühen Neuzeit. In: Augenkitzel - barocke Meisterwerke und die Kunst des Informel, (Ausst. Kat. Kunsthalle Kiel). Hrsg. v. Dirk Luckow. Kiel 2004, 41-45.

Schneider, Sabine und Heinz Brüggemann (Hg.): Gleichzeitigkeit des Ungleichzeitigen. Formen und Funktionen von Pluralität in der ästhetischen Moderne. Paderborn 2011.

Schweizerisches Idiotikon. Wörterbuch der schweizerdeutschen Sprache. Begonnen von Friedrich Staub und Ludwig Tobler und fortgesetzt unter der Leitung von Albert Bachmann, Otto Gröger, Hans Wanner, Peter Dalcher, Peter Ott, Hans-Peter Schifferle sowie Hans Bickel und Christoph Landolt. Bände I-XVI: Frauenfeld 1881-2012, Band XVII: Basel 2015-. Online-Datenbank. 5. November 2020: idiotikon.ch [= Schw. Idiotikon].

Stahl, Enno: Neo Neo Dada. Zeitgenössische Dada-Rezeption bei Mara Genschel, Ann Cotten und Oswald Egger. In: Hugo-Ball-Almanach. Studien und Texte zu Dada. Neue Folge 8 (2017), 125-147.

Vedder, Björn: »Egger, Oswald«. In: Munzinger Online/KLG - Kritisches Lexikon zur deutschsprachigen Gegenwartsliteratur [2014]. 5. November: http://www.munzinger.de/ document/16000000774.

Volkmann, Johann Jacob: Historisch-kritische Nachrichten von Italien, welche eine Beschreibung dieses Landes, der Sitten, Regierungsform, Handlung, des Zustandes der Wissenschaften und insonderheit der Werke der Kunst enthalten. 2. viel verm. u. verb. Auflage. 3 Bde. Leipzig 1777-1778.

Wagner, Monika: Schlitze, Schmutz und Flecken. Ruinenästhetik in Kunst und Mode. In: Bilder, Räume, Betrachter. Festschrift für Wolfgang Kemp zum 60. Geburtstag. Hrsg. v. David Ganz, Steffen Bogen und Wolfgang Brassat. Berlin 2006, 422-435.

Weiss, Christina: Nicht gemacht für lineares Lesen. Über Oswald Egger. In: Sinn und Form 59.2 (2007), 277-281. 
